\title{
A Fundamentally Irreversible World as an Opportunity towards a Consistent Understanding of Quantum and Cosmological Contexts
}

\author{
Helmut Tributsch \\ Bio-Mimetics in Energy Systems Program, Carinthia University for Applied Sciences, Villach, Austria \\ Email: helmut.tributsch@alice.it, www.helmut-tributsch.it
}

Received 20 June 2016; accepted 21 August 2016; published 24 August 2016

Copyright (C) 2016 by author and Scientific Research Publishing Inc.

This work is licensed under the Creative Commons Attribution International License (CC BY). http://creativecommons.org/licenses/by/4.0/

(c) (i) Open Access

\section{Abstract}

In a preceding publication a fundamentally oriented and irreversible world was shown to be derivable from the important principle of least action. A consequence of such a paradigm change is avoidance of paradoxes within a "dynamic" quantum physics. This becomes essentially possible because fundamental irreversibility allows consideration of the "entropy" concept in elementary processes. For this reason, and for a compensation of entropy in the spread out energy of the wave, the duality of particle and wave has to be mediated via an information self-image of matter. In this publication considerations are extended to irreversible thermodynamics, to gravitation and cosmology with its dependence on quantum interpretations. The information self-image of matter around particles could be identified with gravitation. Because information can also impose an always constant light velocity there is no need any more to attribute such a property to empty space, as done in relativity theory. In addition, the possibility is recognized to consider entropy generation by expanding photon fields in the universe. Via a continuous activation of information on matter photons can generate entropy and release small energy packages without interacting with matter. This facilitates a new interpretation of galactic redshift, emphasizes an information link between quantum- and cosmological phenomena, and evidences an information-triggered origin of the universe. Self-organized processes approach maximum entropy production within their constraints. In a far from equilibrium world also information, with its energy content, can selforganize to a higher hierarchy of computation. It is here identified with consciousness. This appears to explain evolution of spirit and intelligence on a materialistic basis. Also gravitation, here identified as information on matter, could, under special conditions, self-organize to act as a super-gravitation, offering an alternative to dark matter. Time is not an illusion, but has to be un- 
derstood as flux of action, which is the ultimate reality of change. The concept of an irreversible physical world opens a route towards a rational understanding of complex contexts in nature.

\title{
Keywords
}

\author{
Fundamental Irreversibility, Gravitation as Information, Redshift as Entropy Phenomenon, \\ Constancy of Light Velocity, Time, Information Driven Universe
}

\section{Introduction}

Conventional physics is based on time invertible natural laws. A recent re-examination of the important and much applied principle of least action however led to the conclusion that the world is fundamentally oriented and irreversible [1]:

"Free energy aims at decreasing its presence per state, within the constraints of the system concerned"

Action, energy times time, is thereby produced and minimized. This is the relevant phenomenon linked to the passage of time. Entropy generation is a fundamental, rate controlling process and has also to be considered in elementary mechanisms. One consequence is that the particle-wave duality had to be formulated differently. Particle (energy $E_{p}$ ) and wave are not equivalent, but the energy in the distributed wave $\left(E_{w}\right)$ is less valuable, because it is highly diluted. Its energy has inferior working ability and is partially present in form of entropy, as non-useful energy $\left(E_{e}\right)$. As a consequence, when a particle is converted into a wave, information (energy $E_{n}$ ) has to be simultaneously provided for the back conversion of the wave into the particle [1]:

$$
h v=E_{p} \leftrightarrow \sum_{w} E_{w}+E_{e}+E_{n}
$$

The particle-wave duality thus becomes a dynamic process, which involves information on matter (symbolically depicted in the center of Figure 1, where arrows indicate energy in form of entropy and the dot pattern energy in form of information on matter, besides of energy in form of the wave).

It could be shown that this concept gives rational explanations for quantization, the double slit experiment, and for quantum correlation. Quantization of electron orbits around a nucleus arises because the energy for information also minimizes and selects the simplest patterns (schematically shown in Figure 1, bottom left)). The recognition of a second slit by a particle proceeding through the first slit works, because the particle dynamically changes into the wave and the information on matter brings this information back to the particle after the slits. Quantum correlation can simply be understood as a consequence of splitting up the information self-image of matter, when particles split up. When information is present, which remains relevant for both separating particles (e.g. conservation of spin), this information cannot split up and remains linking the two separating particles (schematically shown in Figure 1, top left).

Since information on matter (dot pattern in Figure 1 center) has an energy content and should always be around elementary particles with the program to decrease energy per state, it must be detectable and measurable. It could be identified with the phenomenon of gravitation. Obviously this would then be the link between elementary processes and cosmology (indicated in Figure 1, top). Since a fundamentally oriented and irreversible world, with energy tending to decrease its presence per state, also explains the second law of thermodynamics, this is sufficient motivation to explore additional important phenomena in physics. Here the role of entropy for radiation, the law governing self-organization processes, the meaning of time, of gravitation, the interpretation of relativity, and cosmological phenomena will be considered in the light of the proposed paradigm change.

\section{Results}

\subsection{Entropy in Quantum Processes and Cosmology}

The new proposed particle wave duality (Figure 1, center) involves a fundamental consideration of the entropy concept due to spreading of energy into space. This is a nano-scale concession for what is macroscopically known as entropy and dealt with in thermodynamics. Traditional quantum physics has a long history of conflict 


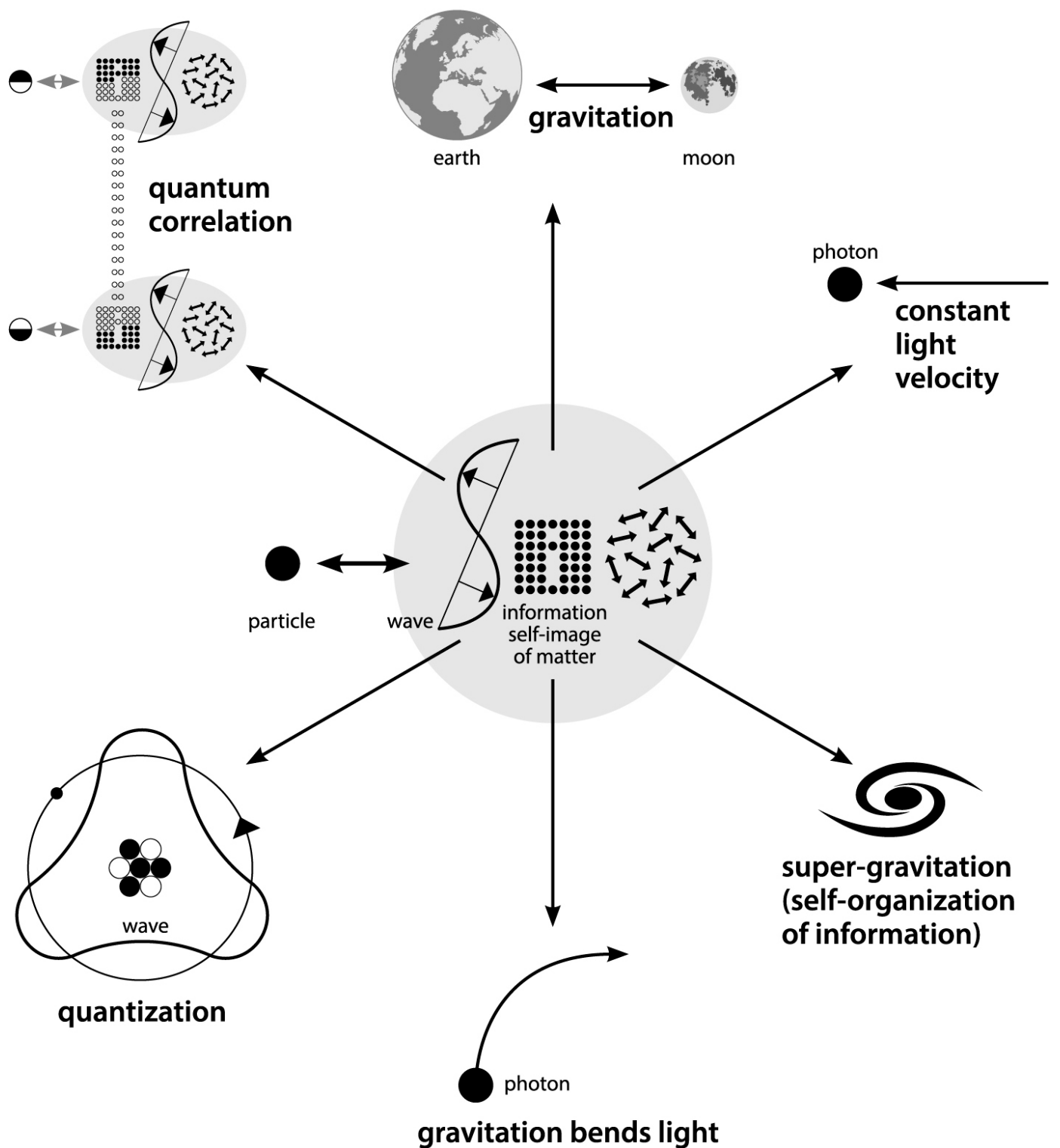

Figure 1. The particle-wave duality in "dynamic" quantum physics (shown in center) considers a fundamental generation of entropy due to spreading energy into space, but needs information on matter (dot pattern in center) to recover the particle. This information self-image of matter is found to be gravitation and to be the key for rationally understanding quantum physics, the always constant light velocity, the link to cosmology and diverse additional natural phenomena.

with thermodynamic interpretations. This was not yet the case at the start of quantum physics. Einstein, for example, used Wien's considerations and his law to argue that the entropy change $\Delta S$ of a radiation field, when its volume is expanded (Figure 2(b)), acts like known for an ideal gas with $E / h v$ as particle number $\mathrm{n}$ [2]. The formula he dealt with reads ( $E$ = radiation energy, $v$ = frequency, $\beta=$ constant, $V_{0}=$ original volume, $V=$ expanded volume):

$$
\Delta S=\frac{E}{\beta v} \log \frac{V}{V_{o}}
$$

as compared to the well known formula for entropy generation by an expanding ideal gas $(n=$ mole number, $R=$ gas constant): 


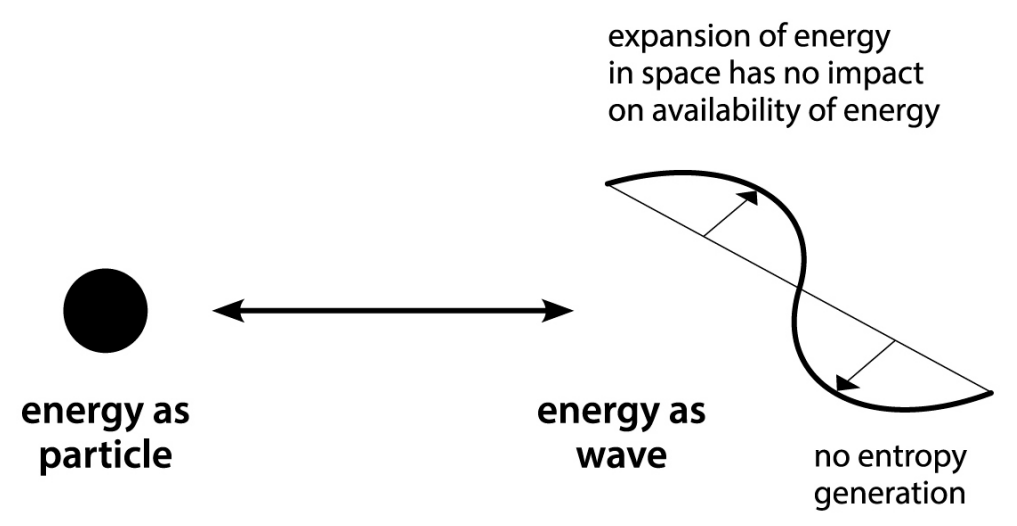

(a)

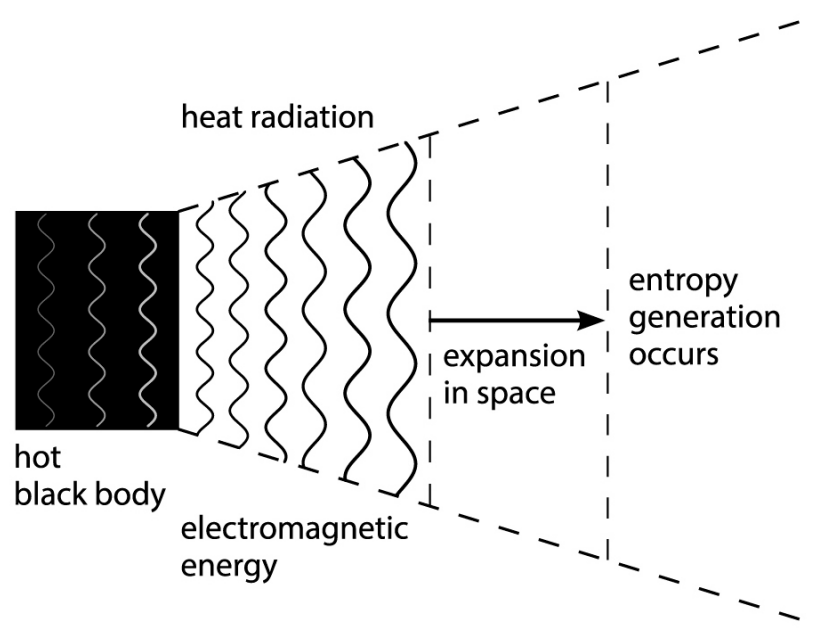

(b)

Figure 2. (a) By stating a dualism of particle and wave (energy concentrated and distributed respectively) classical quantum physics has eliminated the relevance of space for energy (compare the proposed new interpretation in Figure 1 centre); (b) From classical physics it is however known that when radiation expands from a hot black body, less valuable energy in the form of entropy is generated. A shift of frequency and radiation temperature is the consequence (b).

$$
\Delta S=n R \ln \frac{V}{V_{o}}=2,302 n R \log \frac{V}{V_{o}}
$$

The formulas show, that, when radiation or a gas is spreading into an infinite volume, the entropy gain also tends towards infinity. This means that all energy involved in a radiation field (or a compressed gas) should finally be dissipated and converted into non useful, small, chaotic energy quantities of $T \Delta S$. Einstein wrote: "this Equation (3) shows, that the entropy of monochromatic radiation of sufficiently low density varies according to the same law as the entropy of an ideal gas (Equation (4)) or that a diluted solution”.

It is well known that Einstein was awarded the Nobel prize for this finding on the particle nature of photons and its application to electron emission from solids. It is a remarkable science philosophical circumstance that he used an irreversible phenomenon of spreading radiation, entropy generation, to establish a quantum property (later called photon) which then turned out not to tolerate irreversibility and entropy production during its existence. Indeed, in conventional quantum mechanics photons, once emitted into space and spreading cannot generate entropy along their long way by changing frequency and radiation temperature, until they finally interact with matter. During this latter process photon energy is still fully available, as immediately after generation of the photon. In contrast, relation (3) requires, that radiation spreading into space gradually converts its energy in- 
to "chaotic", non useful energy in the form of entropy, radiation of much lower frequency.

An energy loss, observed as a redshift of frequency, is actually observed when photons from characteristic light emission (e.g. from hydrogen) are arriving from deep space. Since a photon without impact can quantum mechanically not lose energy this phenomenon is actually not attributed to entropy turnover, but to a claimed cosmological expansion of free space itself. This (invented) expansion of empty space, as an alternative to not considered and quantum mechanically not tolerated entropy generation by single photons, is expected to stretch the wavelength and is matched to explain the observed redshift.

Here it is stated that fundamental irreversibility requires that photons lose energy during the process of spreading into space. A mechanism must consequently exist, that allows that.

The fundamental irreversibility of radiation processes became already established during a discussion between Planck and Boltzmann from 1897 [3]. The involved thermodynamics of radiation has since been intensively assessed (for a review see e.g. [4]).

For an adiabatic expansion of radiation the following relation is known to be applicable ( $v$ = frequency, $V=$ volume):

$$
v^{3} V=\text { constant }
$$

The frequency of radiation is changing with the volume (a property finally not tolerated for photons in free space by quantum theory). Using Wien's law which states how the frequency $v$ in the maximum of black body radiation is changing with temperature $(T / v=$ constant) one obtains the complementary relation

$$
V T^{3}=\text { constant }
$$

Expansion of radiation into space thus changes radiation temperature (6) and radiation frequency (5). Relation (5) has actually been used to interpret the origin of the $2.72^{\circ} \mathrm{K}$ microwave background radiation from space as a relic of Big Bang explosion, when the universe was assumed to be much smaller. But radiation itself was not understood to expand adiabatically in a given static space to produce entropy (for which the formula was originally calculated), changing radiation's temperature and frequency, and giving rise to microwave radiation as entropy product. In contrast empty space itself was assumed to expand to a large volume, respecting adiabatic conditions, and stretching the wavelength of photons to microwave dimensions [5].

In order to respect quantum theory, which does not allow a photon to generate entropy without collision with matter, and which claims to be complete and not improvable, entropy phenomena are obviously ignored. The observed or expected frequency shift is presently explained via a new and daring theory: that of an exploding universe, which stretches and expands its void [6]. How can a void, originally defined to contain nothing, develop such properties, and where does the abandoned photon energy go?

The problem with conventional quantum theory is fundamental. It cannot deal properly with the role of space for energy and especially with radiation energy spreading out in space (Figure 2(b)). A photon in an expanding radiation field around a light emitting star cannot engage in energy loss through entropy generation.

After emission it simply maintains its energy and can only change it when interacting with matter or with gravitation fields. This already should have indicated that conventional quantum theory is not complete. Such a conclusion is however, as well known, severely contradicted by quantum physicists [7] [8]. The author is convinced that a theory can never be proved to be correct and complete within one and the same system (such as a time-invertible world) (compare the considerations of the mathematician Kurt Gödel [9]). For a discussion of this claim of completeness of quantum theory from a hierarchy above, the choice between a time-invertible and a fundamentally oriented "dynamic" world, compare [10].

The claim, that quantum physics cannot be improved or extended, has thus crucially shaped the present understanding of cosmological processes. In actual cosmology the increasing redshift of starlight from increasingly distant stars is now mostly attributed to the postulated expansion of empty space (and to a smaller extent to the Doppler effect). Doing this astrophysicists essentially ignore entropy generation as described by relation (3). This relation requires that entropy generation by radiation fields expanding into a vast universe exists and is not limited by distance and only restricted by the final turnover and consumption of the available radiation energy. The consequences for the interpretation of the universe are, of course, dramatically different for the two approaches.

Let us use macroscopic, empirical thermodynamics as an approximation to understand photons in a fundamentally irreversible universe. We look at the energy $T \Delta S$ turned over in entropy related processes for individual photons by multiplying relations (3) and (4) with the temperature $T$ and substituting $R$ for $k$, the Boltzmann con- 
stant, in (4). Then $k T$ is the appropriate scaling factor for energy which is relevant for entropy formation. For a typical temperature in space of $2.72^{\circ} \mathrm{K}$ it can be calculated to amount to $0.240 \times 10^{-3} \mathrm{eV}$. This is microwave radiation. When one takes the maximum from the microwave background radiation in space one determines a microwave energy of $0.6 \times 10^{-3} \mathrm{eV}$. A first conclusion is that in an irreversible universe the actually observed background radiation is simply entropy dumped by spreading radiation (what relation (3) requires). A black body distribution of this radiation is actually to be expected when thermodynamic mechanisms of entropy generation are involved. And entropy generation by starlight over billions of years should actually make microwave radiation the dominant radiation in space [11].

In astrophysics the ratio between the frequency of emitted and observed light is described via $\mathrm{z}$, a dimensionless constant. For the microwave background radiation a $z$-value of $z=1089$ has been determined. This means that the change in frequency (energy) from the original photon is 1089 times bigger than the energy of the microwave photon. The highest confirmed redshift of a very distant galaxy appears to be $z=8.55$. This means that the arriving photon has only conserved $11.6 \%$ of its original energy. This is entirely consistent with a thermodynamic interpretation of an expanding radiation field. Radiation is emitted and generating entropy according to relation (3). A frequency shift is occurring (relation (5)). But since very far galaxies are still visible only part of their radiation energy has been converted into microwave radiation in form of entropy. Or alternatively: they are still visible, but red-shifted, because not all visible radiation has been converted for entropy.

However in the present picture of an increasingly expanding universe the concerned galaxy is seen to flee at an extremely high speed. There already seems to be a serious problem with transgression of the speed of light, especially when considering galaxies at opposite ends of an exploding universe. However the well known (but not easily comprehensible) explanation of present cosmological physics is that this is not the case since the empty space itself is being stretched and expanding so fast so that the galaxies are just (passively) moved that way.

The here discussed "dynamic" quantum physics derived for a fundamentally oriented world can consider energy turnover for entropy production, when radiation is expanding into space. This is possible for two reasons. First because "a system tends to decrease its energy per state", a conclusion drawn from the principle of least action (statement (1)). And second it is possible, because the information self-image of matter is continuously mediating the interchange between the particle and the wave $\left(E_{n}\right.$ in relation (2)). Thereby it already considers the entropy of the expanded energy of the wave and may also consider the entropy requirements of a photon in an expanding radiation field.

The photon is consistently reassembled from the wave state and thermodynamic law may be imposed via information, which could then release not any more available, entropic energy in form of low energy radiation. This is graphically shown in Figure 1, center, where the equivalence between particle and wave within "dynamic" quantum physics is explained. Information on matter is mediating between a wave plus the entropy involved in spreading energy out into space. This information on matter could be programed in such a way as to consider thermodynamic law, which demands that the radiation itself, when spreading into space, generates entropy. How could this happen? How could distribution into space be monitored by a photon? It could happen via gravitation, because information on matter, as involved in the dynamic particle-wave duality, can be identified with gravitation (see Figure 1, center and top, and later in more detail) and because this gravitation is equally a determining cosmological factor.

In "dynamic" quantum processes photons can consequently lose energy via generation of entropy. They are behaving as required by thermodynamics and expressed by relation (3), which Einstein used to argue for the existence of photons [2]. This way, in principle, Einstein also pointed at an irreversible nature of photons. The emitted, not any more useful energy for entropy is, as above estimated, very low (lower approximately by a factor of 1089). Because of the fundamentally claimed minimization of free energy content for the photon (1), because of the small amount of energy for entropy turned over, and because of conservation of momentum, an (entropic) energy release without change of photon direction is to be expected. This way the here proposed thermodynamic process of entropy generation has nothing to do with the already long ago rejected idea on "tired" light, caused by hypothetic collisions of photons with dust in space [12] [13]. No blurring of starlight would be seen and the light particles from the outmost detected galaxies would already have lost 88,4\% of their energy for entropy production, for the liberation of low-energy microwave quanta. This is compatible with thermodynamic law for entropy production of spreading radiation (3) which allows in principle all radiation energy finally converted into non useful, chaotic energy, $T \Delta S$, if the space covered by spreading radiation approaches very large 
values (since the logarithm in (3) tends to infinity).

The irreversibility of expanding radiation is here a very important argument. It should therefore also be derived from additional evidence. It is well known that from an expanding gas energy can be recovered via a piston in a gas motor. The same can be imagined to occur with an expanding radiation field, if energy is harvested via a sun sail (Figure 3). This also characterizes the expansion of light into empty space as an irreversible, entropy producing process. Part of the energy of expanding photons (the energy which could have been harvested by the solar sail) is not any more available.

The information on matter, dealing with the dynamic particle-wave duality $\left(E_{n}\right.$ in (2)) considers and deals with the entropy production due to the expanded wave. And it could also consider entropy generation due to radiation expansion from a star or a galaxy. A key for this is the identity of gravitation, information on matter, on quantum level and cosmological level. An interaction of a photon's information on matter (identified with gravitation) with gravitation in space towards implementing a fundamental empirically confirmed law is to be expected. The increasing redshift of starlight from increasingly distant light sources in space may thus be considered to be essentially the result of entropy loss and the microwave background is simply the expected entropy dump. Unexplained redshift patterns, such as different redshifts from quasars within galaxies, or periodical redshifts, have now to be considered to be essentially a problem of information handling in nature. In this context also the question, whether light from individual galactic objects is beamed in certain directions or evenly distributed will have to be considered (it affects $V$ in relation (5) and may explain why light sources in similar position show different redshifts). Significant research efforts will, of course, be needed to learn much more about this natural information technology. In conclusion: before speaking about an exploding universe, an inflating space by stretching the void, and a dramatic flight movement of galaxies, entropy loss by spreading photons has to be understood and considered.

Basing on such conclusions we should interpret Hubble's empirical law accordingly and differently. Mathematically, this law describes the supposed increase of the escape velocity of galaxies (in kilometers per second) with respect to our earth and is presently the key for describing the dynamics of the universe including the so-called dark energy. It describes the galaxy velocity $v$ via the Hubble constant $H_{o}$ times the distance $D$ of the galaxy from earth in light years $\left(H_{o}\right.$ expressed in kilometers per second per megaparsec, which is 3.26 million light years. Its value is estimated to be between 50 and 100 , mostly at 70 ):

Galaxy velocity: $v=H_{0} D$

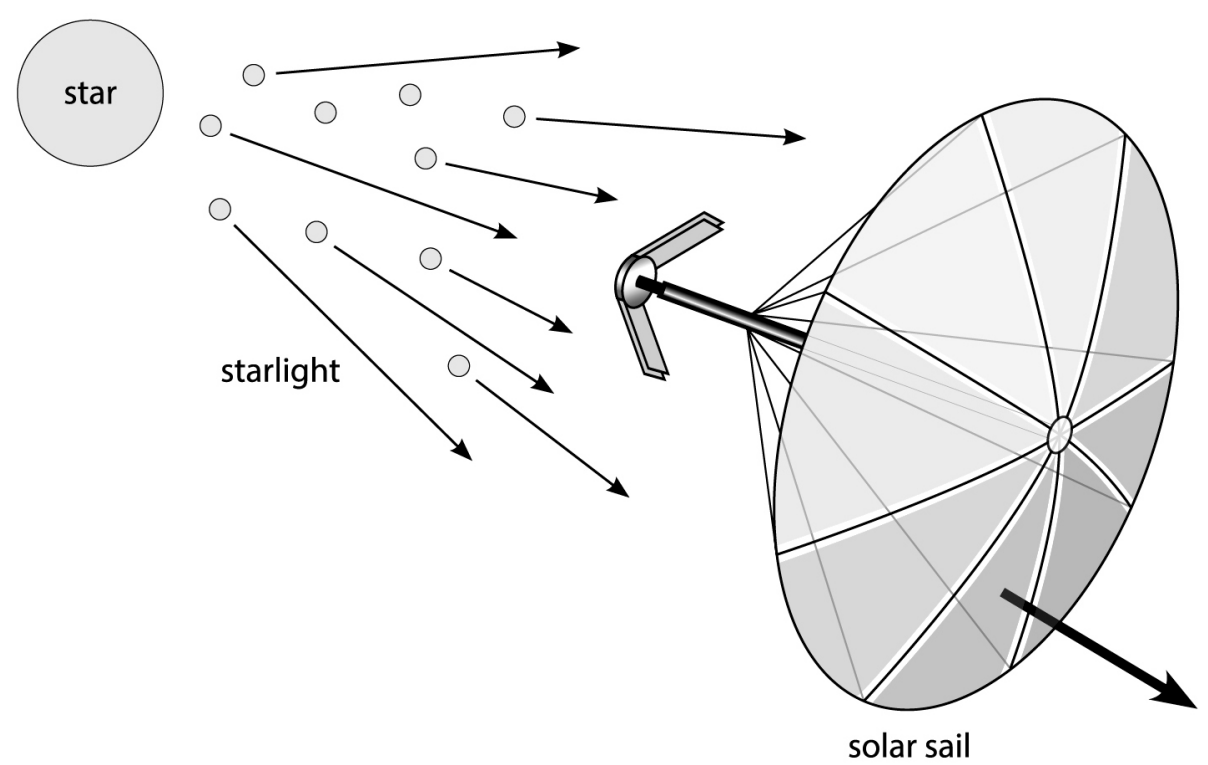

Figure 3. When starlight spreads into empty space, entropy is generated. A solar sail accelerated by starlight could harvest part of this spread-out energy. But when this energy is not harvested, it is converted into a non-useful, "entropic" form. "Dynamic" quantum physics allows interpretation of entropy generation as red-shift, conventional quantum physics adopts an inflation process of empty space as alternative explanation. 
This empirical formula can, just by mathematical transformation, be converted into a formula expressing the new interpretation of the redshift. If we divide the formula left and right by a distance (kilometers), which is mathematically allowed, we arrive at a statement on the frequency $v$ ( 1 divided by seconds) of light from galaxies in dependence on their distance from earth $D$. This way the formula is still valid but allows an entirely different interpretation. The new proportionality factor should now be named $F_{o}$ and has the dimension of frequency shift per mega-parsec (3.26 million light years):

$$
\text { Photon frequency: } v=F_{o} D
$$

The energy contained in a photon is proportional to the frequency $v$ and a frequency shift per distance (in mega-parsec) consequently reflects energy loss via entropy turnover $\Delta S$. The proportionality factor would be related to the entropy turnover: $F_{o} \approx \Delta S$. This means that the frequency shift of light from galaxies changes with their distance $D$ from earth and is dependent on the rate of photon energy loss $F_{o}$ via entropy production.

The latter, entropy production, is not a constant but would be expected to change logarithmically with the distance from the light source and would also be affected by the geometry of light spreading. This simple adapted formula explains, without assuming an inflation of space, why our earth appears to be the center of space. In fact it is of course not the center, but we are seeing from all directions incoming light, which has lost valuable energy on the long journey from galaxies in space for entropy production. We are seeing entropic energy loss corresponding to the distance covered. And we understand why the microwave background radiation is quantitatively the most important radiation source in the universe. It accumulated over billions of years (in contrast to being considered a short snapshot of Big Bangs optical echo).

\subsection{Are Uncertainty and Zero Point Energy Fundamental?}

There is an important quantum phenomenon, which has changed the present way of recognizing and understanding nature, since it is now accepted as fundamental. It is described by the uncertainty relation, which was first proposed by Werner Heisenberg. It cannot directly be derived from fundamental principles but results from the empirically grown considerations on quantum physics. The uncertainty relation states that position and momentum as well as energy and time of a quantum object cannot be measured exactly, but only with an uncertainty, which is proportional to Planck's constant of action (h). Concerning the energy-time uncertainty an interpretation is that when the time interval is too short, energy cannot exactly be determined. For a measurement of frequency (energy) several cycles have to pass. The average energy of an outgoing photon shows a certain distribution, a line width. Fast decaying states have a broad line width, slow decaying states have a sharp line width. Since energy and mass is related, the mass of the photon is accordingly more or less accurately defined. Why can the mass (energy) of a photon, $E_{p}$, vary?

It turned out that the uncertainty is not merely a simple observer effect, but a fundamental property of quantum systems. Uncertainty became a mysterious basis of quantum theory, which thus adopted an element of unpredictability. Not the reality of particles is described in quantum mechanics, but our statistical observation and measurement of these particles. It is known that Einstein was not amused by such a perspective and is known to have commented: "God does not play dice”. But the uncertainty relation has influenced our present view of the universe: The zero point energy and the possibility that energy and particles are popping out from nothing are consequences drawn from the uncertainty principle. Such concepts have been applied to Big Bang scenarios and to Black Hole interpretations.

How can a game with dice be understood within the "dynamic" quantum understanding? Does this empirically deduced quantum phenomenon really mean a departure from causality and rationality? Let us start with the "dynamic" interpretation of the particle-wave duality from (2) and find out whether we can rearrange the relation to understand uncertainty. One can bring the time from the frequency $v=1 / t$ to the right side and multiply the result with the particle energy $E_{p}$ in the numerator and denominator to get a relation with the formal structure of the energy-time uncertainty:

$$
h=E_{p} t \leftrightarrow\left(\sum_{w} E_{w}+E_{e}+E_{n}\right) t \frac{E_{p}}{E_{p}}=E_{p} t \frac{\sum_{w} E_{w}+E_{e}+E_{n}}{E_{p}}
$$

$E=E_{p}$, the total energy of a photon, multiplied by $t$, the time of its existence in a given state, is proportional to $h$, the Planck constant of action. What is interesting now is the proportionality constant, which shows up on the 
right side of Equation (9). It is the ratio between the energy of the particle and the energy of the wave (made up here of distributed energy, entropic energy, energy of information). Considering the dynamic nature of the particle-wave duality in a fundamentally oriented world this is not really a constant equally to one. The magnitude of the proportionality constant will simply vary and depend on whether, during the instance of measurement, the energy is more present in the particle or in the wave. This suggests that the uncertainty is related to the intermediate presence of energy in form of a wave (which also conventional quantum theory assumes) and more specifically, in the form of information on matter for the reconstruction of the particle from the wave. It may be that, depending on the snapshot of inter-conversion between particle and wave captured during the measurement act and related to the presence of information, fluctuating and partial particle properties are reconstructed from a wave. Here, the information self-image of the particle $\left(E_{n}\right)$ enters and plays an important role. Under given conditions of measurement only part of the photon mass may be reconstructed. $E_{p}$ in relation (9) is simply affected by the ratio between wave and particle state. An uncertainty is to be expected. But there is no reason at all to assume a fundamental origin of uncertainty and unpredictability. There is a complex mechanism involved, which is determining quantum phenomena. The origin of uncertainty is here entirely understandable. It is linked to the structure of the dynamic particle-wave duality. The proposed process of inter-conversion between particle and wave is responsible for a statistically determined reconstruction of the particle's properties. It is true that a detailed theory for our interpretation of the particle-wave dualism still has to be developed. A deterministic statistical chaotic mechanism as a reason for the observed uncertainty is to be expected, since matter in an irreversible world is interpreted as self-organized energy (see below and [10]). Self-organized energy and matter can be subject to deterministic chaos, especially when given structures are decaying or being transformed. And, which is most important, deterministic chaotic statistics cannot, when experimentally observed, be distinguished from arbitrary statistics [14].

Within a "dynamic" quantum physics there is consequently no reason to assume a deviation from causality and determinism. The uncertainty phenomenon is merely shaped by a complex natural process including information turnover. With its complexity it is veiling a logical physics behind.

It is well known that the uncertainty relation predicted that a zero-point energy remains when all other energy is removed from the system. An absurdly large amount of such zero-point energy should consequently be available all over the universe.

The particle-wave duality introduced for a time-oriented physics (2) has shown the information self-image of matter $E_{n}$ to be the relevant factor for eliminating paradoxes from quantum physics. The difference in lowest energy between quantum and classical system, which zero point energy appears to be, should therefore also be related to the information self-image of matter. And indeed, when the energy of the wave ( $E_{w}$ and $E_{e}$ in (9), middle) is set zero, the information self-image remains in the formula. The zero-point energy or vacuum energy (in quantum field theory) may not be pure energy itself, but information on energy (and matter). It can be identified with gravitation (see later for more details), which indeed has a cosmological significance. The zero point energy has actually been related to the cosmological constant [15]. Information on matter (gravitation) appears to be the key for understanding the interrelation between (here deterministic) quantum uncertainty behavior and cosmological parameters.

\subsection{The Law Governing Irreversible Thermodynamics}

It has already been mentioned, that the second law of thermodynamics, the observation that entropy maximizes in a closed system, can directly be derived from the basic statement of irreversibility (1) [1]. Traditional physics, basing on time-invertible fundamental processes, cannot do that, which is a significant drawback.

Far from equilibrium self-organization occurs, which requires the involvement of feedback processes, which need a "before" and an "after". In a fundamentally irreversible, time oriented world self-organization is a straightforward phenomenon. It is the domain where life or hurricanes are functioning. Here, a maximum entropy production is to be expected. Such energy systems, powered by a through-flux of energy, will transform all available energy by decreasing its presence per state (relation (1)) and generating entropy. Entropy production will be rate controlling and only be limited by the constraints of the system.

On the basis of empirical observations a similar result, maximum entropy production for self-organized systems, was already obtained by other scientists. But they could not derive it from more fundamental considerations and prove it. A maximum entropy production rate in the atmosphere, as a state determining the climate, has 
been proposed by Paltridge [16] and later others [17]. Pioneering work on maximization of the entropy production rate in relation to human ecology came also from Swenson starting from 1988 [18].

This principle of maximum entropy production, derivable from empirical observations, and following here from the "dynamic" energy postulate, has, however, found strong opposition. It was argued by John Ross from Stanford University that the rates of chemical reactions are controlled by (non-vectorial, non-oriented) thermodynamic quantities such as Gibbs free energy and activation energy and not by the rate of entropy production [19]. And in consequence, he speaks of the "invalidity of the principle of maximum entropy production". The criticism expressed by John Ross is perfectly reasonable, but it is reasonable only on the basis of reversible thermodynamics, its non-dynamic quantities of state and its traditional understanding of kinetic mechanisms. This situation is entirely changed with the here advanced proposal to introduce a dynamic property of energy via a paradigm change (relation (1)). When energy is given the property or tendency to decrease and minimize its presence per state, then it simultaneously drives the rate of entropy production and controls it. This leads directly to maximum entropy production far from equilibrium within the constraints of a given system. The criticism forwarded by J. Ross [19] no longer applies and, in fact, the requirement mentioned by him exactly matches the introduced paradigm change. The free, available energy no longer "sleeps" but drives entropy production. The law:

"Self-organized processes develop maximum entropy production within the constraints of the system involved"

for the non-linear range of irreversible thermodynamics is a straightforward consequence of the "dynamic" energy proposal. It is supported by a great deal of empirical evidence [16]-[18]. It explains, for example, the destructive potential of atmospheric storms, which can rise to dramatic proportions of hurricanes within one day or two. Since biological systems and societies are self-organized, this finding has also significant consequences for understanding biological evolution and the future of human civilization (for a more detailed discussion of this complex subject see [10]).

\subsection{Gravitation: Information Self-Image of Matter}

Within the here presented new understanding of the particle-wave dualism (relation (1) and Figure 1, center) energy in the form of particles tends to dilute itself in space, forming a wave and generating some entropy. But information (with energy $E_{n}$ ) is activated to keep this process in balance. All together should have the ability to act towards a decrease of energy per state. Compared with the classical picture of matter, there is a difference. To understand matter rationally, it is now not sufficient to describe matter, particle or wave. One also has to consider information on this matter. And this information should be real and somehow present and observable. What is it? This information self-image has an energy value and should therefore somehow be measurable around matter. What energy-related phenomenon is found around matter, increases with matter and penetrates matter? The answer is obvious. It must be gravitation. Can macroscopic gravitational energy indeed be related to this information-mediated balance between concentrated and diluted energy within a dynamic particle-wave duality?

There is an obvious analogy to be seen in our information system, based on television, cell phones and navigation systems. Here the information is contained in the electromagnetic waves, which are transmitted to be present in the environment and to function via digital signals. As with gravitation, the distribution is not even. The communication-mediating electromagnetic waves provide energy, and transmit information. The information, which is considered as mediating activity between the particle and wave nature of matter must also be measurable via the energy, which it involves. Gravitation, the mysterious force around matter, indeed involves energy, gravitational energy. It is therefore concluded that it is actually gravity, which is equivalent to the energy of information $E_{n}$ (Figure 1, top).

The dynamic energy initiative has yielded a new interpretation of gravitation! It is not a force in the classical sense, but information with a well-determined aim, namely the aim of decreasing the energy per state. But it acts like a force, but unlike other forces (electrostatic, magnetic) only in direction of attraction (and a decrease of energy per state). This deserves being explored more in detail, since gravitation is known to be something very special and a very weak "force". It also offers an interpretation of gravitation, alternative to that generated by the four dimensional space-time in the general theory of relativity.

When the information self-image of matter, besides of reconstructing the particle from the wave, aims at de- 
creasing the presence of energy per state, what happens when one attempts the opposite, to increase the energy per state, for example via acceleration of the same mass? One would expect a "counterforce", a force that attempts to prevent an increase of energy per state. It can only be inertia. Gravity and inertial forces are determined to be proportional to the same mass or energy. They are forces active towards a decrease of energy per state or are activated in the case of violation of such a situation. The equivalence principle, the observation that inertial and gravitational mass are identical, appears to follow in a straightforward way. The information self-image of matter, interacting with mass and energy, is doing the job. It is gravitation and acts on the particle while the particle decreases its energy per state. It is equally gravitation, which should matter, when the particle's energy per state is forced to increase during acceleration and thereby generates inertia, a counterforce. This conclusion results from the identification of the information self-image of matter with gravitation. The gravitation on the location concerned should, in fact, be the collective gravitation from the mass of the universe. It is also responsible for inertia. This supports quite a vague idea by Ernst Mach on the origin of inertia, which was later discussed by Einstein, but only partially applied in the general relativity theory.

In the general theory of relativity the phenomenon of gravitation is explained differently and is much more complicated. The mass of a body generates a curvature of space-time. When moving along such a curved space-time, gravity and inertia are expected to become identical for a mass. The experimentally verified equivalence principle is a basic claim, which had been introduced into the general relativity theory. Space was adapted to act in such a way that the equivalence principle is fulfilled.

What is, in fact, gravitation and what speaks for its identification with the information self-image of matter in the model presented here? In science today gravitation is still a poorly understood phenomenon and is discussed in a conflicting way, both physically and philosophically [20]. The "Grand Unifying Theory", GUT, for elementary particles cannot adequately assign it, because it seems to be something special [21]. It will therefore be all the more interesting to find out what the dynamic energy theory has to say in particular about gravitation as an information self-image of matter.

Gravitation works for large masses of matter, but equally for elementary particles. But the difference in magnitude is large. Between two neutrons and between two weights of one kilogram the ratio of attractive gravitational forces is estimated to be of the order of $10^{54}$. Gravitation is nevertheless present everywhere. Gravity indeed supports the tendency to decrease the energy per state (relation (1)). It attracts matter and thereby decreases its potential energy and generates non-useful, chaotic energy, for example, heat. When a rocket accelerates at 9.81 meters per square second, an astronaut feels gravitation like on the earth's surface. He feels inertia, the resistance against an increase of energy per state. He feels it like a person on the earth's surface feels gravitation, the pressure to decrease energy's presence per state. When the rocket engine stops, the inertial force disappears. The energy per state does not increase any more. But if this pressure towards a reduction of energy per state exists why are atoms then not totally compressed? Why are the negatively charged electrons and the positively charged cores of atoms maintaining a large empty space between them? There is an obvious reason why gravitation does not compress atoms and eliminate the enclosed empty space [1]. Quantization results from this attempt, since also energy for information (gravitation) gets minimized. This way electrons are forced into well-defined diffuse orbits of standing waves, which correspond to minimum energy-information conditions. Equation (2), by the way, would then describe the relation of gravity to quantum theory (it occurs via $E_{n}$, the energy involved in information). It would describe a straightforward interrelation between the gravitation phenomena in space and the function of atoms and elementary particles (compare Figure 1). Such a relation has been intensively searched for in conventional physics but has never been found. The field of quantum gravity deals with that problem, aiming at a "theory of everything". String theory is an example of approaches, which have been followed. They seem to have basically failed up to now, and from my point of view the reasons are obvious: quantum theory has to be modified to become "dynamic" and the gravitation concept of the general theory of relativity has to be challenged (see later). This appears to be a harsh statement, but is a necessary consequence of the paradigm change discussed here.

Within the model analyzed here the unification of gravitation with quantum physics is a side product, however with an important new insight: the energy of information, $E_{n}$, identified with the energy contained in gravity causes quantization to occur [1], but is itself not quantized. Also the mysterious phenomenon of quantum correlation would be related to properties of gravitation and would suddenly become more transparent. Since the particle-wave duality involves an information self-image, this self-image has to be dealt with when the particle is split up (Figure 1, top left). Due to conservation laws only part of the original self-image can be split up and the 
rest will maintain joint information for the separating particles. Lacking a detailed theory it can presently, however, not be said up to which distance such information contact will be supported. However, for energetic reasons, I predict the reach of quantum correlation will not be infinite, as quantum physicists today claim, for quantum theoretical reasons. All together the interpretation given here for quantum correlation would not contradict logics. Correlated particles are acting like couples which maintain a reasonable contact via a cellular phone even when temporarily separated locally. One just has to be prepared to accept that information on matter is part of quantum reality, and information (gravitation) also involves the possibility of communication between separating particles.

The fact that this very special phenomenon of quantum correlation can readily be explained is considered to be strong support for the discussed dynamic energy approach. Indeed, the very circumstance that quantum correlation exists also justifies the existence of an information self-image of matter for the original, undivided particle (Figure 1, center). And this information on matter is exactly what makes up the hitherto mysterious gravitation phenomenon.

In contrast to other forces in nature gravitation penetrates all matter. A material body can also not be extracted from a gravitational field nor can it be shielded from it and gravity cannot be neutralized. It can, however, be partially or totally overcome by inertia. A high flying airplane in a sharp curve downwards leaves its passengers temporarily without gravitation. Within the model presented here this means that the drive to decrease the energy per state, and to give by to the attraction of gravitation, is just compensated by the drive resisting against an increase of energy per state, within the phenomenon of inertia. These two opposite drives are felt as forces, which in the described situation just compensate. In fact, however, these are two different communications of information, which are just compensating and neutralizing each other.

Gravitation itself is caused by mass. Gravitation is, therefore, not the consequence of interaction of masses and it could until now not be explained on a more fundamental basis, which however the present effort claims. Gravitation needs mass, but it is not a consequence of action. Since action is the consequence of a cause, which requires time, gravitation should not be exposed to the flow of time. This is, in fact, also in agreement with the new model provided, which identifies gravitation with information. During the information-mediated particlewave interchange no energy is converted and consequently no action is generated. Therefore, no time flows. In contrast, matter and energy themselves are exposed to changes and time. It is information and the energetic aspect of information which aims at decreasing the energy per state. In a gravitation field this is equivalent to an attracting force and explains why there is no repulsive counterpart of gravitation. It gives weight to objects, attracts them and causes them to fall towards the ground. From the time of Newton we know that when two particles with the mass $\mathrm{m}$ interact, the gravitational force between is proportional to the square of the mass divided by the square of their distance. According to Newton it is a force at a distance, but nobody understands how such a far-reaching force may fundamentally work.

For Einstein, in his theory of relativity, gravity is not a typical force, but the consequence of the movement of objects through a curved space-time. It is an interaction at close distances. In fact, within such a picture, acceleration arises from a "curved" time. This is a time, which changes its flow in such a way as to simulate acceleration. One should reflect here for a moment and try to imagine how a void with nothing in it can have such an elaborate property of manipulating time, which not even the most sophisticated technology can do. Nevertheless, the reasons for planets cycling around the sun are consequently not attractive forces, but a curved space-time structure around the sun and the planets. The energetically permitted orbit for a planet is then its essentially elliptical planetary orbit. Photons, light particles, are also characterized by a mass and momentum and consequently deviated by gravitation. Einstein has simply transformed all the mystery about gravitation into a sophisticated four-dimensional space-time. All moving objects should be exposed to the same natural laws, including a constant light velocity. But Einstein's theory of general relativity cannot explain the fundamental character and mechanism of gravitation. Empty space is just manipulated to impose the phenomenon of gravitation when masses are present. However, nobody can explain why and how empty space can implement such very special properties and this is a significant weakness. From the point of view of understanding nature, it is a clear setback. No detailed rational theory is imaginable that can explain such complicated behavior of empty space. As mentioned, it is so complicated that Einstein himself, years after publication of the general relativity theory, was again speaking of an "ether" with special properties that should be able to do the job [22].

The dynamic energy approach discussed here which has already helped to eliminate diverse paradoxes and irrationalities offers a much simpler alternative theory for gravitation: the energy related to information, $E_{n}$, in- 
volved in the interplay between particle and wave with the aim of decreasing energy per state. Information tells masses how to act. They act like systems guided by remote control. The mechanism is not action at a distance like Newton's gravitation, and not action at a close distance like Einstein's gravitation. It works as long as information is around and this means here gravitation. The technology works as when information is used to operate a system via remote control. An information signal (gravitation) must reach the moving object. Planets around the sun or satellites around the earth would thus follow the natural "remote control" mechanism operating via gravitation, forcing them to always fulfill the condition of minimum energy per state or, equivalent, of least action. They are exposed to gravitation and, accordingly, select their specific orbit. Wherever a planet or a satellite goes, the condition of a minimum of energy per state is fulfilled. It is ultimately the "dynamic nature" of energy, and its implementation, which is the secret of gravitation. Gravitation is neither a force, nor an interaction. It is information implementing a fundamental natural law, which dictates a decrease of energy per state. Looking at the example of a stone rolling down a hill, it can be said that gravitation forces the stone to always fulfill the condition of minimizing the presence of energy per state via its information content. We know now that we can also say that the stone follows the principle of least action in a dynamic way. The same happens with a satellite circulating around a star or a planet.

Since understanding gravitation is so important let us use an additional check-list of properties of gravitation to verify in more detail whether these can reasonably support the claim raised that we are dealing with an information self-image of matter.

1) "Matter and gravity cannot be separated". This is evident from relation (2) since energy of information mediates between particle and wave form of matter

2) "Shielding matter from gravity is not possible. It penetrates all matter". There is no contradiction to be expected to this finding.

3) "Gravity effects can accumulate to large phenomena". There is no reason why energy of information should not accumulate in the presence of large quantities of matter. Information is additive or, if oriented into the opposite direction, subtractive.

4) "Gravity only attracts, it does not repel". This is evident since only attraction of matter can lead to a decrease of energy per state (relation (1)). Such a property makes gravity different from typical forces.

5) Gravity and inertial forces correspond to each other, but are opposite in direction. They reflect the action of masses from the universe. A mass feels all the gravitational forces of the universe. If a mass is accelerated, it acts against the gravitational forces of the universe. This is what the Mach principle claims and thereby rejects an absolute, empty space. In our picture, the following phenomenon would happen: when matter accelerates, the energy per state would increase, so that the energy of information, $E_{n}$, would counteract. This would be experienced as inertia. The effect would be supported by all masses from the universe felt via gravitation. Inertia would thus simply be a counteraction to a violation of gravity, which (here) implements the claimed tendency of energy to decrease its presence per state (relation (1)).

6) If gravitation is not the consequence of a cause, then it is not exposed to a flow of time. Indeed, the dynamic particle-wave duality expressed in (2) does not involve a time flux, since energy only spreads and contracts in space, but is not turned over. It is an on-going, eternal phenomenon and will be present as long as matter exists. As a consequence, there cannot be a spreading of gravitation when mass is localized, since spreading would involve time.

7) The general theory of relativity predicts gravitational waves. Lately scientists claim to have detected them [23]. The identification of gravity with information mediating the particle wave duality allows a clear conclusion: information, and the energy related to it, is not expected to be able to generate waves itself. However, self-organized information, expected in a region of exceptionally high gravity and additional energy, could, in principle, induce oscillating phenomena (see next subchapter). But also bursts of information on matter could be liberated via nonlinear mechanisms from cosmic areas with intense gravitation and energy. It should be emphasized here that in order to really confirm an oscillation of space-time according to the general theory of relativity, a parallel oscillation of "time" should be observed. It should actually be detectable since, according to Einstein, time is what the watch shows.

8) In a given gravitation field all bodies, whether as light as a feather or as heavy as a hammer, are exposed to the same acceleration ("g" on the earth surface). They approach the ground at identical speed if air is not present to exert different friction. One can rationally understand that the information self-image imposes such behavior, an identical acceleration. The information given in the same gravitation field towards a decrease of energy per 
state is simply identical for different mass objects. The force experienced (and the weight of a body) is, of course, different because acceleration has to be multiplied by the mass, which is accelerated, to obtain the force. The acceleration due to gravity on the surface of the moon is only $0.16 \mathrm{~g}$. Correspondingly less information on matter is active there towards a decrease of energy per state. The acceleration towards a decrease of energy per state is apparently changing proportional to the density of information.

The information self-image of matter could thus indeed be the origin of what is called gravity. It is not a force and not an interaction. It is information on the state and dynamics of matter, energy, and implements a fundamental dynamic law. This turns simultaneously out to be a reasonable and interesting explanation of gravitation. One understands why it had to become a relevant property of nature.

If we, for example, want to understand the deviation of light in a gravity field we would have to explore interaction possibilities of the particle-wave relation (2) with the perturbing systems. Tentatively I would say that, when gravitation (information) from a photon superposes gravitation (information) from a larger mass, a corresponding additive superposition of information should be expected. Light will consequently react in response to external gravitation (information on matter). A quantitative theory remains to be developed.

Gravitation also plays a fundamental role in determining the dynamics of the universe. It may consequently be expected that the information involved in gravitation acts in a similar way in large scale dimensions. It is information, which should consequently control generation, evolution and fate of the universe. Within some kind of fractal similarity, which could be assumed, there should consequently also exist an information self-image of the universe (Figure 4). It is information, an information self-image of the primordial world, which activates useful energy at the start of a re-born universe, which then again aims at generating non-useful, chaotic energy in the form of entropy, by allowing energy to decrease its presence per state.

A fractal link between quantum processes and cosmic events (Figure 4) implies a joint fundamental (mathematical) basis, which may also help explaining how spreading photons can adequately consider space for entropy generation. This way it is possible to confront the Big Bang theory, which is characterized by extreme and partially irrational assumptions and many contradictions [24], with a fractal model for the evolution of the universe, which emphasizes the role of information. This SI-universe (Self-Image universe) is based on a (periodical) recreation of the primordial universe through an information self-image (Figure 4) (for more discussion of this subject compare [10]). The idea that information should be active at the beginning of cosmic development is not alien to the human intellect and human imagination. The Bible, for example, says: "In the beginning was the word" [25]. And such an information induced start of a free energy rich universe with subsequent increase of entropy and local growth of order at the expense of entropy production is entirely in agreement with our understanding of thermodynamic laws. And from the impressive function of 3D printers we understand how information can indeed create structural and dynamic reality. The information based self-image universe is a high-tech scenario compatible with presently recognized and understandable natural laws and with working information technology.

This is in marked contrast to the Big Bang scenario where the universe starts from nothing to chaos and maximum disorder. Here a creation of energy and time-flow from nothing has to be postulated, as well as an explosively fast expanding, inflating void. The latter incredible postulate is in fact needed to explain the reasonably high uniformity in space. But it is also needed to increase the potential for additional disorder (for the purpose of allowing entropy formation during energy conversion processes) via a huge added abstract volume (created through inflation of the void, the empty space). This should enable the energy conversion processes that generate the observed structures and function. The Big Bang theory describes a low-tech model, on the basis of very speculative additional assumptions.

\subsection{Consciousness, Spirit and Super-Gravitation}

Natural science has recently been criticized for not being able to explain evolution and function of consciousness and human spirit. Philosopher Thomas Nagel [26] claimed, that the preconditions for evolution of spirit, in terms of physical laws, must have been there in nature before humans developed. His argument has to be taken seriously. Humans have developed consciousness and there may be innumerable living beings in the universe with similar abilities.

In a fundamentally oriented and irreversible world there is a straightforward answer to this important question: consciousness can be explained on purely materialistic, scientific basis. It can be understood in the following way: 


\section{. \\ photons, elementary particles}

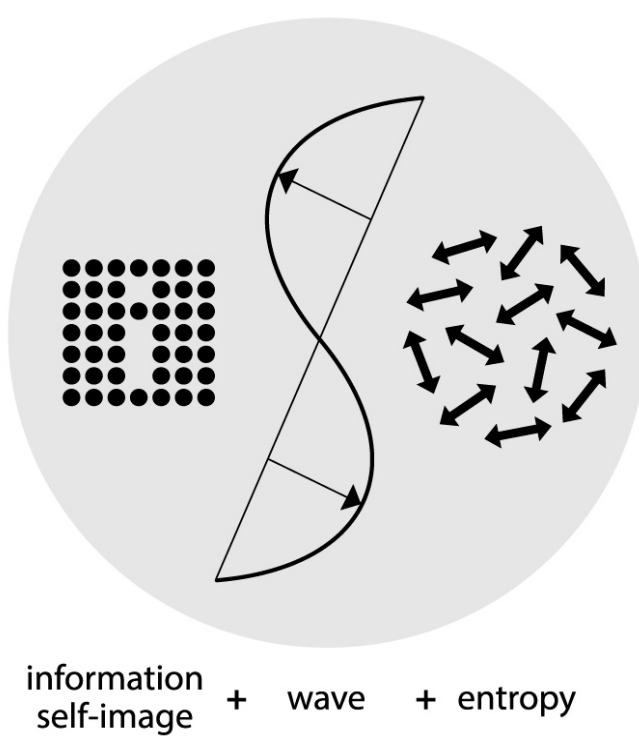

(a)

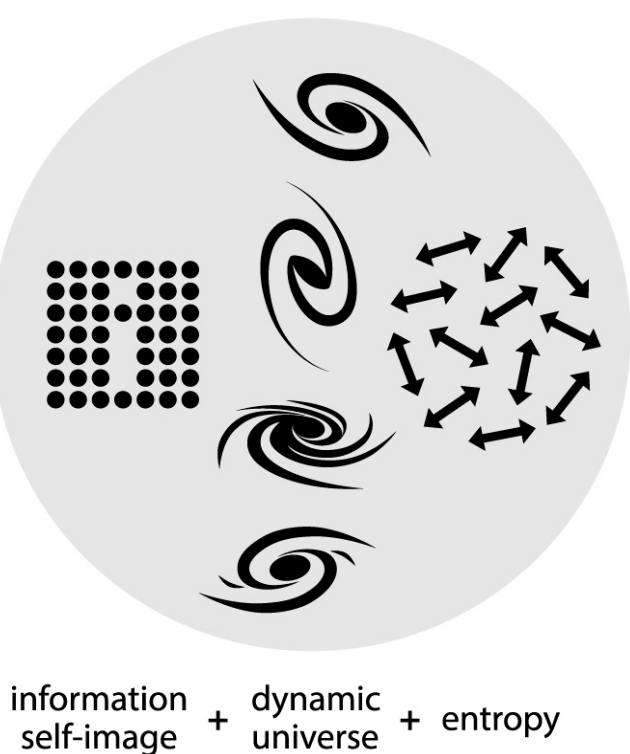

(b)

Figure 4. Within a fundamentally oriented reality the universe has apparently a fractal, selfsimilar structure and function: the gigantic space seems to work as a "Self-Image Universe" (b), essentially analogue to the proposed sub-microscopic "dynamic" particle-wave dynamics (a). In both cases information is set aside for the final recovery of the worn-out or spread-out product of energy activity.

In a fundamentally irreversible world in which time is proceeding (via a flow of action) there is a "before" and an "after". Feedback processes are possible. Therefore self-organization of matter will be a straightforward consequence, as long as energy is flowing through the system, and the system is pushed sufficiently far from equilibrium.

When looking at biological life-forms, one, of course, recognizes that self-organization, $S O$, can occur with matter. Self organization of matter is consequently a function of matter $m_{t}$. which can be formulated as follows:

$$
S O=f\left(m_{t}\right)
$$

Mathematically, matter can consequently be handled in such a nonlinear way that self-organization works and 
can be calculated and demonstrated. Matter is something that occupies space and has mass. Matter is consequently a function of mass $m$. Self organization, $S O$, therefore proceeds with mass:

$$
S O=f\left(m_{t}(m)\right)
$$

Mass is, as well known, mathematically linked to energy via $E=m c^{2}$. Consequently, energy can, in a fundamentally irreversible world, also self-organize, when appropriate conditions exist.

$$
S O=f\left(m_{t}(m(E))\right)
$$

What can be the consequence of self-organization of energy? It is proposed, that the large family of elementary particles is the product of self organization of energy (in more detail discussed in [10]). Their formation and interaction, in nature, and their generation, during high energy experiments, would have to be described and discussed accordingly. Mechanisms and properties of self-organized processes would have to be considered. The conversion of structure and function of one particle into other particles or from a photon particle into a wave could, for example, be triggered just by a small parameter change.

How can now information be considered for self-organization?

Since information, $I$, has an energy content and is a function of energy, energy can be expressed as a function of information. This can be considered in (11c), so that information should also be able to self-organize, when appropriate conditions prevail:

$$
S O=f\left(m_{t}(m(E(I)))\right)
$$

Therefore information itself will also be able to self-organize, provided the system supplies essential material properties and is pushed sufficiently far from equilibrium. In addition adequate structures must exist and adequate feedback processes between information modes have to occur. This important conclusion follows from these simple mathematical considerations. They show, that from the fact, that self-organization of matter exists, self-organization of information can be deduced mathematically.

As matter (energy), which brought about life, can self-organize, information, which is also based on energy turnover, can, in principle, do the same (Figure 5). The preconditions only are that some degree of order in information structures has to evolve before and that the information system has to be pushed sufficiently far away

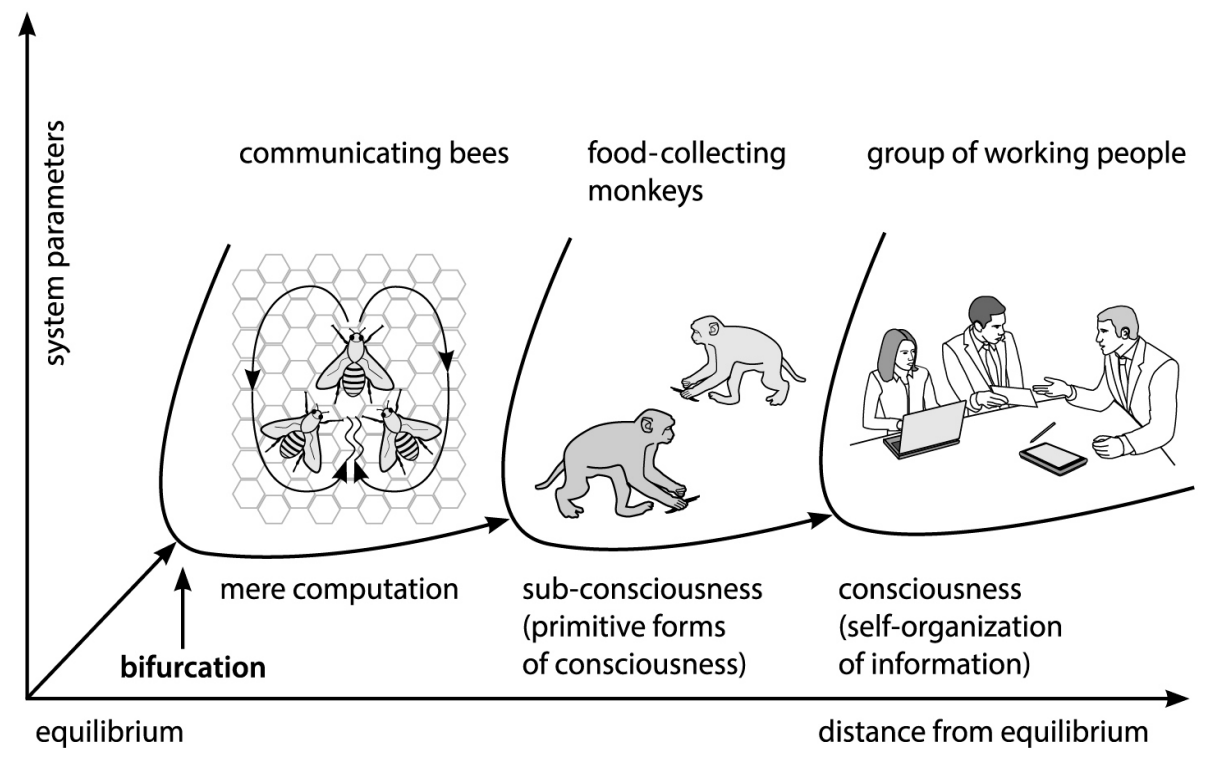

Figure 5. The diagram shows the influence of the distance from equilibrium for information systems, which is controlled by the through-flow of energy. During evolution more and more energy became available to the brain and shifted information activity from mere computation to self-organized computation, which initially corresponded to more primitive forms of subconsciousness, and finally yielded consciousness. 
from equilibrium. Also a flexible, variable hardware seems to be required. The information storage elements involved in self-organization could be chemical, as implemented in the genetic code, or electrochemical, as functioning in our brain. The result would be a much higher hierarchy of computation. This higher hierarchy of consciousness is a fact. In addition it is clear that we are actually dealing with an information system, and that consciousness has gone through evolutionary steps (sub-consciousness, collective consciousness, personal consciousness). Furthermore it is known that there is high energy consumption going on in the brain. An additional evidence for such an interpretation of consciousness is the above calculated relation between information and self-organization (11d). All this speaks for a really functioning mechanism of self-organization of information. A science based on equilibrium, time-invertible laws and a time-neutral energy phenomenon could not support such a process, and has for that reason been criticized [26]. The implementation, within the dynamic energy concept in an oriented world, of a fundamental energy-time drive is decisive for the shift of a system away from equilibrium and for the necessary feedback processes.

Consciousness, as humans evolved it, is a very sophisticated information program. It gives a person a personality and active control over his situation. The theory that consciousness controls our computing brain via self-organization of information is also supported by additional arguments: Kurt Gödel [9] developed a theorem in 1931, the incompleteness theorem, which became one of the most important statements of modern logic. It can be interpreted to state that a machine controlling another one has to be associated with a more superior hierarchy. The comparison of self-organization of information in relation to information itself with a living bacterium and its chemical components in water gives the adequate answer: a living bacterium functioning via its self-organization structure represents a hierarchy clearly above that of an aqueous solution of all chemical substances, which make up the bacterium. Its living activities also clearly demonstrate that it can control chemical activities.

Self-organized information could therefore in principle well control pure information. It is hierarchically definitively placed above pure computation. It can, therefore, be understood as the mechanism of consciousness. It makes man conscious and helps him to organize his life in a complex environment in a more creative and competent way. It also shapes his intentions and determination. The "dynamic energy" approach allows a purely materialistic interpretation of consciousness.

An interesting additional subject for further thinking on self-organization of information is also gravitation. In present physics gravitation is, as above mentioned, still a mystery and has been discussed as a very strange force. But in this study gravitation is found to be information, mediating the particle-wave duality (relation (2)) and implementing a decrease of free energy per state. This kind of information, gravitation, should also be able to self-organize, provided enough energy is turned over to push the gravitation system far from equilibrium and feedback processes can develop. A significantly higher and more elaborated gravitation, a kind of super-gravitation may be the consequence (indicated in Figure 1, right, bottom). And super-gravitation should also have the potential to become spatially structured like living organisms. This phenomenon may, for example, take place in the center of galaxies where high concentrations of matter and energy are present and interact. But it may also occur more widely distributed over the space, structuring it via gravitation patterns. Astrophysicists know a phenomenon called "great attractor". It attracts galaxies, including our own. This may be the result of self-organization and structuring of gravitation. The resulting super-gravitation will have much more sophisticated properties with respect to the interaction of matter than ordinary gravitation. Above all it is to be expected that the capacity to decrease the energy per state, which this information—gravitation—should implement, will be much more effective. There should be a much higher attraction between matter, and it is perfectly understandable why light can penetrate a super-gravitation zone, though with deviations due to the mirage effect, which is actually observed. This happens like an ordinary mirage on earth develops under a special weather condition, a condition of the atmosphere, which itself is a self-organized phenomenon. Self-organized gravitation in space could indeed be compared to special weather conditions on earth. Both self-organization phenomena may generate optical mirages.

Obviously self-organized gravitation is an alternative theory to dark matter since it would explain a high and spatially structured gravitation, which is not balanced by sufficient visible matter. But no additional matter would be needed in this case. There would not be a need to search for invisible new particles, but there must be sources for energy turnover in the environment to push the gravitation system far from equilibrium for self-organization. It is obvious that such an explanation, together with the above, alternative interpretation of galactic redshift as entropy phenomenon, requires a corresponding new evaluation of cosmologic dynamics. 


\subsection{Relativity Theory and Four-Dimensional Space-Time: Do We Need Them?}

The information self-image of matter ( $E_{n}$ in (2) and dot pattern in Figure 1, center) provides a straightforward and rational explanation for gravitation on quantum and cosmological level in an irreversible natural environment (see above). The same information self-image of matter could also guarantee an always constant light velocity in any reference system. The generation of a constant light velocity of a photon particle could simply be part of the program executed for recreating the particle from the wave. The program should be the same for every reference system. That this is indeed possible regardless of varying velocities of different reference frames is visualized in Figure 6. Imagine an airplane in full flight receiving digital information from a distant television station. A perfect television picture would be seen. In fact, if a corresponding information would be transmitted to the plane for driving a three dimensional printer, this would also be possible irrespective the relative velocity. The printer could produce a toy-car, which is able to drive at a constant velocity. In the same way information could also be used to regenerate a photon from the wave (relation (2)) with always the same constant light velocity in the given reference frame. Information would have been used to produce an object able to propagate

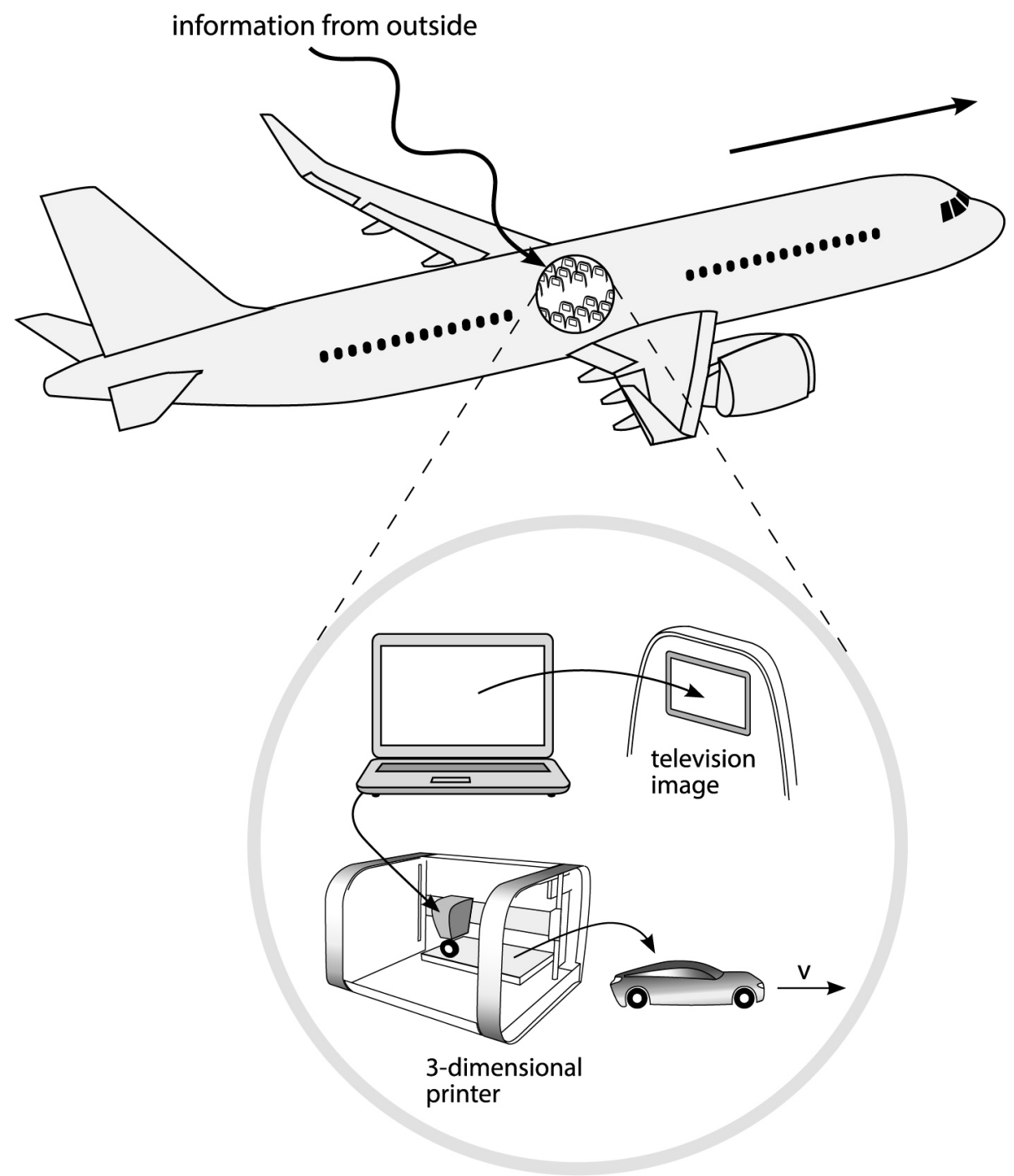

Figure 6. Why can the information self-image of matter reconstruct light particles with an always identical velocity? It is like transmitting a television program or the information for a three-dimensional printer to a flying airplane. The printer could produce a toy car, which then drives with a given velocity. The outcome is fixed and will be identical irrespective of the airplane's flight direction and speed. Information transfer is the key for rationally understanding constant light velocity. 
with constant velocity, regardless the flight direction and velocity of the plane or a reference system. This would be a rationally understandable, working mechanism for the fundamental implementation of an always constant light velocity.

The information self-image of matter can indeed explain both gravitation itself (as shown above) and the always constant light velocity (indicated in Figure 1 right and top). Can this route of explanations replace relativity theory?

The special and general theory of relativity has originally been designed to explain these two natural phenomena: the always constant light velocity and the strange force, called gravitation. The empty space was mathematically manipulated in such a way that gravitation, inertia and the always constant light velocity are adjusted and function as experimentally observed. A complicated four dimensional space-time resulted which around matter shows a dramatic curving of space and time. The general relativity theory has, even though it is based on time invertible basic laws and does not consider energy conservation, become the standard for explaining highly dynamic space phenomena, ranging from the assumed Big Bang event to space inflation and black holes. Why has the general theory of relativity become so important in explaining gravitation and the dynamics of our universe? First, nobody had an idea of how to explain an always constant light velocity differently, besides of suggesting a special "ether", which was the model preceding Einstein's special relativity theory [27]. In addition, the mathematics of general relativity turned out to be so complex, that it was only gradually understood [28].

Many of its solutions turned out to be irrelevant, others difficult to interpret. Then new circumstances entered. Since the availability of modern computers, physicists can more easily play with the complicated formula for general relativity and space-time involving ten parameters [29]. Via these many parameters and the singularities, which the general theory of relativity yields, such as extreme peaks and deep valleys, an incredible amount of possible scenarios can be described. Only few solutions seem to make sense. There is presently no indication as to why the relatively inaccurate predictions of the general theory of relativity cannot be explained by other, less farfetched and less complex theories. But what about the famous relation $E=m c^{2}$ derived by Einstein from the relativity theory, which describes the energy of a mass $m$ at zero velocity. It may be necessary to say here that it has been shown that the derivation of this formula was not really based on logic reasoning but already presupposed in the derivation of the result [30]. Classical derivations of analogue formulas have also been cited [31]. Einstein speculated that the energy formula he derived for light (and which included arbitrary choices) may also be valid for any energy in general. Two years before Einstein an Italian geologist, Olinto de Pretto, published the same relation between energy and mass deriving it from other non-relativistic considerations [32].

The point here is that we want to understand what this formula actually means within the relativity theory. In fact the formula $E=m c^{2}$ has nothing to do with relativity. It would be still valid if the four-dimensional space did not exist. Einstein had neglected relativistic considerations prior to obtaining the famous energy-mass formula. It can be derived with classic arguments only. The energy-mass relation can therefore not be used in support of the four-dimensional space-time concept. This is here an important argument.

In addition, the reasoning developed here within a time-oriented physics, requires that mass is self-organized energy (see dicussion above and relation (11c)). When this is accepted to be true, then mass is accordingly also in this picture proportional to energy. The proportionality constant $\left(1 / c^{2}\right)$ is, however, not evident and is expected to be much more complicated. The fact that the energy-mass relation also follows from the dynamic energy approach via the interpretation of mass as selforganized energy is an additional support for its consistency.

The concept of a fundamentally irreversible world has thus yielded an entirely new and unexpected explanation for gravitation and the always constant light velocity. Explaining gravitation, inertia and constant light velocity via information is rational, in contrast to giving such properties to a manipulated empty space itself. What incredible properties of empty space must be imagined to control constant light velocity or to adjust the time flow around masses to mimic inertial movements! Relativistic changes in length and time, which are predicted by the relativity theory, can be considered to be simply measurement phenomena caused because of the finite light velocity. Measurements from different orientations and velocity frames would produce different values of length and time. A really shrinking rocket would simple dismantle, but theory predicts it could later land regaining its full dimension.

Also the energy measured from different orientations and velocity frames would produce different values. Can an individual energy system simultaneously have different energy values? What about experimental "proofs" 
for relativity theory? Presumed gravitation waves measurements and gyroscopic measurements involve tiny experimental effects (stretching the space by one part in $10^{21}$, or by the width of an atomic nucleus for detection of gravitation waves; gyroscope spinning angle change of 1.8 thousands of one degree per year). The here discussed physics of a fundamentally irreversible world could provide alternative explanations. The focus of attention should be the action of information on matter (and gravitation respectively). It explains light velocity, gravitation and inertia in a different way and could consequently also explain phenomena attributed to them differently and rationally.

The here advanced concept of information involved in elementary particle properties and gravitation has the potential to make quantitative predictions, provided a more detailed quantitative theory is elaborated along the line of thoughts presented here. To give an example: In the case of photons deviated by gravitation it is the information self-image of matter, which interacts with information in form of gravitation from other sources, while reassembling of the photon from the wave occurs. This superposition of information on matter will generate the observed change in the flight trajectory of the photon. It is expected that a four-dimensional space-time is not needed for explaining the phenomena observed. Information could much better interpret them than claimed irrational properties of free space (for a short summary of presently favored concepts on space, time and gravitation see for example [33]). And it would be intuitive understanding, like we understand e remote controlled device responding to an external signal.

\subsection{The Flow of Action and the Phenomenon of Time}

For science today time is an illusion. What time should one take, if time flow for systems in relative movement is variable? Historically numerous personalities have reflected about the meaning of time and contributed interesting information and ideas without really fully answering the question: what is time? [34]-[41]. On the basis of an analysis of the principle of least action it was found that this principle describes a fundamentally oriented world and a fundamental, rate controlling flow of action during energy turnover [1]. This required a paradigm change from a time invertible world to an irreversible one. It means that the free energy of physical systems tends to decrease its presence per state. Consequently production of action, energy times time, is the essential basis of changes. Around us many flows of action are proceeding wherever energy is being converted. This is what causes the observed "time flow" in nature. It is what we see happening in the environment and this is what occurs in our brain. But time itself, as described by " $t$ ", is only an ordering parameter. It has no material or energetic substance at all and can therefore not directly be measured. Clocks determine it nevertheless by measuring the flow of action resulting from an energy converting mechanism. They basically divide it by the energy turned over and calibrate it with a selected periodical astronomical event in days, hours, minutes and seconds.

Time flow, as determined by action, energy times time, itself is not an illusion. When action is transferred to frames with different velocity it stays actually invariant, even within the theory of relativity. In order to determine real time flow in an irreversible world, action has to be transformed to another frame in relative motion, and the time $(t)$, the ordering parameter, has to be determined there by dividing action by the energy turned over locally. Since action stays invariant during transformation, time for energy converting systems would then be the same in frames with different velocities.

This is of course different with time in the theory of relativity. It is separated from energy and transformed independently like energy itself. Here time is relative in frames with different velocities and special phenomena such as time dilation and time travel become possible, which can lead to well known irrational contradictions with physical laws and with thermodynamics. The time " $t$ " in the theory of relativity, which is already separated from substance and energy, is thus a theoretically manipulated time and refers to systems, which do not turn over energy, such as quantum states and elementary particles in such situations. Time delays in systems at different velocities are actually measured (such as with muons) and can be considered real, since they are actually determined. But my interpretation is that it is the measurement process, it is the finite light velocity, which is signaling a distortion of reality. This can be recognized, when several observers at different positions and velocities are measuring the time on an identical reference object. It will be different for each one and only be seen in direction of the relative movement. An observed object cannot simultaneously have different times. Another example is the change of length of an object seen on frames with different velocity. If it would be real and if the object would be a rocket, it would be dismantled during the change of dimension. However, when the rocket is landing, its original size appears re-established. The relativistic phenomenon here is just an illusion of measure- 
ment, based on transmission of information by light. Time in its naked form, $t$, time which the clock shows (according to Einstein) and which is not characterized by substance, matter or energy, is just an ordering parameter in combination with the constant light velocity. In this combination, with the dimension of distance, it evidences the distance across which action can travel in time. But itself this naked time " $t$ " is not able to express action, energy times time, which is the origin of real time flow, as it is expected to drive real phenomena like the Big Bang or Black Holes, which are calculated, using the general theory of relativity. Already for this reason the predictions of the general theory of relativity therefore appear not to express the reality of an energy converting universe. But they can, of course, support imaginative theories due to extreme singularities and many adjustable parameters.

In conclusion: the flow of action, as considered in the principle of least action, is the real, energetic basis of time flow. If we select a machine (e.g. an hourglass), which is providing a constant flow of action, a flow of "least" action in the form of trickling sand, and if we calibrate this flow with an astronomical flow of action (e.g. the movement of the earth around the sun) then we get a "time", which is not an illusion. But the basis of time flow is always the flow of action, and only action, energy times time, should be transformed to another reference with different velocity, if "time" should stay relevant for energy converting systems. However, for exactly such reasons time travel, which is predicted by the theory of relativity, will remain an illusion. "Time" in form of a flow of action is not an illusion and existed and will exist as long as energy conversion occurred and will occur.

\subsection{Black Holes and Quasars: Understanding Space Objects in an Irreversible Universe}

A fundamentally oriented and irreversible world will follow its special laws. Since a time arrow, based on the flow of action, exists, self organization processes will proceed and systems will develop and compete towards maximum entropy production.

Here, an example should be discussed to understand such behavior in relation to the expected dynamics of black holes, in contrast to traditional understanding.

Within the general theory of relativity contracted masses can drastically deform space-time to generate a black hole with an event horizon, beyond which there is no escape for matter and energy. But close to the event horizon virtual fluctuations may cause particle-antiparticle matter to appear and liberate a radiation called "Hawking radiation”, which can gradually lower the mass of the black hole [42]. Time invertible physics of relativity and quantum theory are thus presently used to "understand" the dramatic dynamics of black holes [43].

The approach of fundamental irreversibility draws a different picture since self-organization of matter and energy, as well as maximization of entropy production will become corner stones of understanding. Black holes themselves, which compact and convert large masses through gravitation (here information towards decreasing the presence of energy per state) run gradually into a problem. On one hand they develop to become self-organized systems, but because of the high gravitation, they cannot get easily rid of entropy in the form of chaotic waste energy. The black hole will temporarily serve as a grave for entropy, because gravitation is too high. It would be like if a vegetarian dinosaur could not get rid of digested food any more. How can a black hole deal with such a situation? In this context quasars are especially interesting objects and are typically found associated with a black hole in the center of galaxies with a bulge. This combination could explain how a black hole finally gets rid of its entropy, which becomes confined by massive gravitation. The black holes collect matter and star fragments and rotate them in a so-called accretion disc (Figure 7). From there matter can fall into the black hole or be radiated away with total intensity amounting to between one million to one billion times our solar radiation. In fact, radiation intensities equivalent to that of one hundred Milky Way galaxies have been estimated [44].

Such radiation emitting systems are called quasars. They belong to the most brilliant objects in the universe.

In our view quasars somehow originate from black holes, which have drifted further and further away from equilibrium because of the problem they have in getting rid of entropy due to high gravitation. The quasar black hole assemblies seem to have reached an evolution state where maximum energy turnover and, correspondingly, maximum entropy turnover is approached by a self-organized inorganic system. A black hole alone would, because of extremely high gravity, have difficulties in emitting radiation and matter. But evolution within an irreversible universe has facilitated a way out by modifying the self-organized system while increasing its distance from equilibrium. This way it is probing new "bifurcations" and gradually changing the black hole into a system, which can be understood to be an association with a quasar. The result, a self-organized system with much 


\section{maximum entropy production}

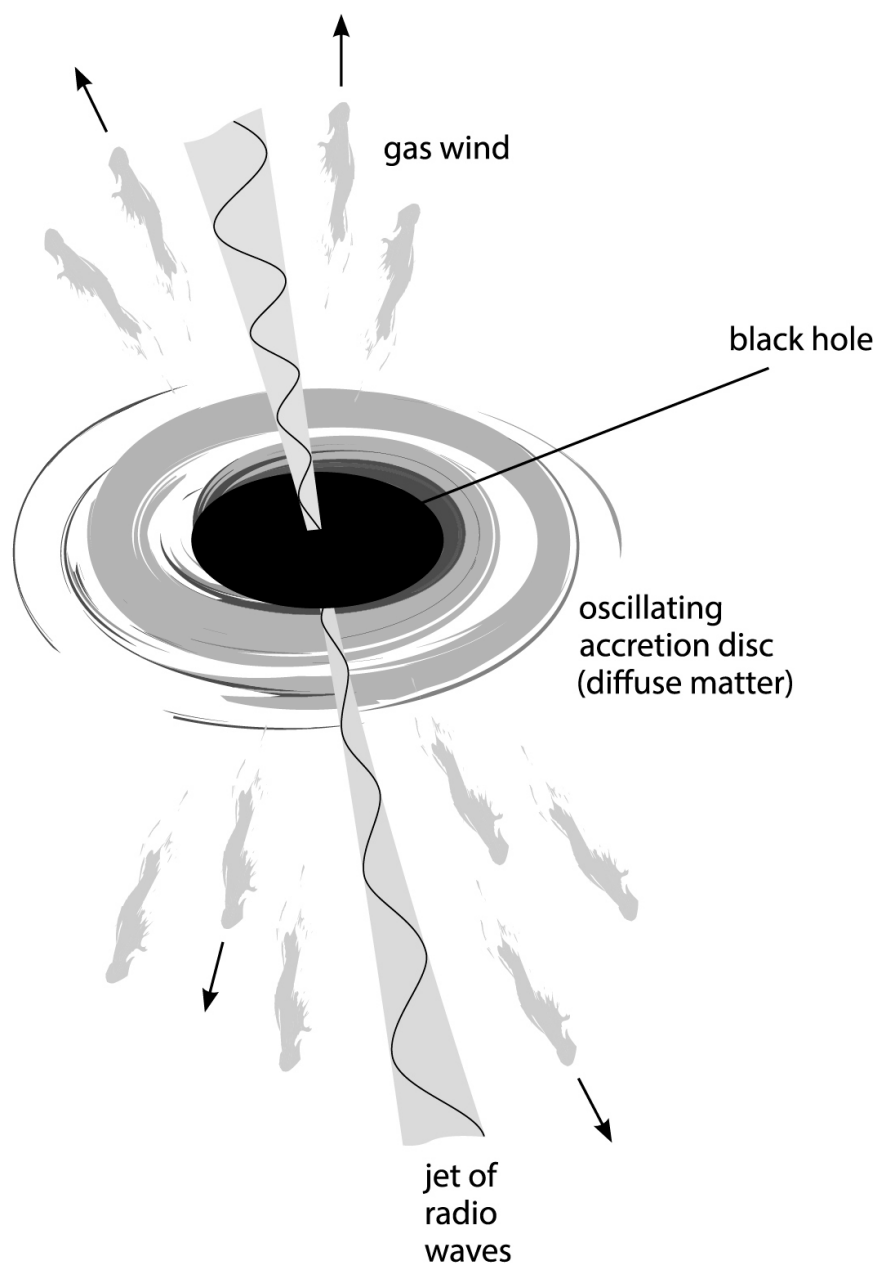

Figure 7. Association of black hole and quasar facilitating maximum entropy production. Both are dynamically self-organized systems, which, depending on the energy turned over, vary their distance from equilibrium and their parameter constellations. Maximum entropy emission occurs through the accretion disc, which appears to serve as a kind of logistic platform for the collection of useful matter and emission of waste energy. Self-organized and spatially structured gravitation (information) may be part of the mechanism.

improved ability for entropy production and emission, is convincing. Also self-organization of gravitation (information, as discussed above) may occur leading to structural patterns with gravitation-free channels for entropy emission into space. Jets of electromagnetic waves are actually seen to leave quasars as well as gases.

Quasar-black hole associations change in light intensity within weeks or months. They are inorganic systems essentially behaving like living animals. I compared them with vegetarian dinosaurs. Their activities depend on the existing constraints. Dinosaurs also generated less entropy when finding and digesting less food.

In this way quasars appear to be associated with black holes to which they donate matter and from which they subtract "degraded, chaotic" energy in the form of radiation and dust. The association between a black hole and a quasar thus testifies for maximum entropy production during conversion of solid matter into liberated waste energy.

The "evaporation" of matter from quasar-black hole aggregates via nuclear processes aims at generating a maximum distribution of "chaotic" energy in space and time. They are basically executing, to a maximum performance, because of optimized constraints, what I claim to be a fundamental law: energy's tendency to decrease its presence per state in space and time (relation (1)). 
In conventional cosmology the role of the quasar is interpreted differently: it is just a secondary phenomenon. Its enormous light emission is expected to result from the accretion disc, where matter rotates while waiting to be accepted by the black hole. Energy is essentially thought to be liberated through friction. In contrast, our interpretation here is that evolution of an inorganic self-organized quasar-black hole system has optimized energy turnover for maximum entropy production within the constraints of the system. It is more or less behaving like a storm that grows into a super-hurricane, distributing its entropy over a wide area. Energy and matter is simply following a natural law, maximum entropy production far from equilibrium (statement (10)). And there is an evolution of energy conversion activity: The developing self-organized black hole system dynamically restructures and "searches" for a way to get rid of entropy. This search is simply the consequence of the recognized fundamental energy property. Entropy confined into a limited space has a higher value of energy per state so that black holes grow. Larger black holes finally find a way to get rid of energy in the form of entropy, chaotic waste energy. They do it by restructuring in such a way that entropy, non-useful energy, can be ejected. This apparently happens via the so-called accretion disc, which forms around the black hole and handles the transport of matter-energy to the black hole and of entropy, non-useful matter and energy, from the black hole (Figure 7).

That the accretion disc is a product of dynamic self-organization is not only evident from its spiral structure, but also from quasi-periodic oscillations, which have been observed to occur in these.

Processes far from equilibrium are expected to control many aspects of space structures, ranging from galaxies of different shapes to aggregations of them showing complex order in form of clusters and lines, but also respecting voids. Gravitation has long been recognized to be a very important parameter for structure and function of space. Here identified to represent information on matter and able to self-organize it plays an even more important role in determining evolution, structure and dynamics of the universe. A remarkable result is that one is dealing with information, which is not only responsible for quantum phenomena, but also for cosmological contexts (Figure 1). This opens new ways for asking questions and reflecting about the origin and destination of the universe and its structures. An information based alternative to the presently favored Big Bang scenario of cosmic evolution becomes evident, as discussed in context with gravitation (subchapter 2.4, for more details see $[10])$.

\section{Discussion}

This effort to explore natural phenomena from the viewpoint of a fundamentally oriented, irreversible world, motivated by a re-evaluation of the meaning of the principle of least action [1], has yielded quite remarkable results. Without additional assumptions, besides of the claim that free energy decreases its presence per state (relation (1)), which led to a dynamic interpretation of the particle-wave dualism, rational explanations for relevant natural phenomena could be obtained. Important is the finding that perturbing counterintuitive assumptions, paradoxes and consequences of presently favored theories based on time-invertible physics, can essentially be avoided. This may be not only because the world is really irreversible, but also because the introduced paradigm change opens the opportunity to consider entropy formation on a fundamental basis. It is possible in form of a reduced energy value when energy is diluted in space. It is for this reason that a wave is not equivalent to a particle within the particle-wave dualism, but information has to be provided to concentrate the energy again in a particle (Figure 1, center). The particle-wave duality is therefore mediated by information on matter. This information has an energy value, is located around matter and penetrates matter. It was identified to be the phenomenon of gravitation. This concept not only allows avoiding quantum paradoxes [1], but also facilitates new quantum interpretations when applied to cosmic contexts. While conventional quantum theory does not allow photons in a spreading radiation field to lose energy for entropy without interaction with matter, this is possible within the dynamic quantum interpretation. The information self-image of matter continuously reassembles the photon from the wave and may adjust the energy balance for entropy generation fulfilling a fundamental law. Since a fractal interrelation is seen between information handling in quantum processes and in cosmological context (Figure 4), a joint functional (mathematical) basis may be assumed, which enables a photon to "sense" the space for considering the entropy law (3). A Hubble-type of equation for the galactic redshift is provided (relation (8)), which explains the redshift phenomenon via entropy production, avoiding the presently favored assumption of a universe inflated by an expanding void. The microwave background is interpreted as entropy dump. Because entropy turnover is a thermodynamic necessity for expanding radiation fields, a quantum physics that can comply with it brings a definitive advantage compared with the established theory postulating an ex- 
pansion of empty space and a stretching of wavelengths as explanation for a cosmological redshift.

Another aspect is thermodynamics itself. The second law of thermodynamics, which claims attainment of a maximum entropy situation in a closed system, immediately follows from the dynamic energy claim (statement (1)) or, equivalent, from the identified meaning of the principle of least action [1]. Since entropy production is rate controlling, and feedback processes are facilitated in a time oriented world, self-organized systems far from equilibrium will aim at maximum entropy production within the restraints of the systems. This is the reason why quasars, in association with black holes, are reaching such a high energy turnover. Terrestrial examples are hurricanes, which, under appropriate conditions, can also approach maximum entropy production and can grow from simple storms to super-hurricanes within days. Since living systems are also self-organized, biological evolution and evolution of human civilization have to be looked at in an unconventional new way (this subject is discussed in some detail in [10]).

A remarkable additional finding is that the information self-image of matter, needed for the dynamic particle-wave duality, can be identified with gravitation. In this way quantum phenomena and cosmological phenomena are linked in a straightforward way. It became clear why gravitation is a special "force". It is actually not a force, but information, aimed at decreasing energy's presence per state.

Because information has an energy content and energy is related to mass and matter, information can also self-organize under appropriate conditions and create a higher hierarchy of information handling. Information, associated with life, when sufficiently pushed away from equilibrium, may therefore self-organize to yield consciousness and spirit on purely materialistic foundation. Consciousness within human personality actually exists. But a time-invertible physics, which defines the flow of time via statistical probability, could never explain such a phenomenon. When information can self-organize, gravitation can also do it, since it is understood as information on matter within the dynamic energy approach. A precondition is that additional energy is turned over and the system is shifted sufficiently far from equilibrium. This may yield a kind of super-gravitation, a gravitation, which is occupying a hierarchy above normal gravitation. It could explain, what is now attributed to black matter, much more intensive and also structurally patterned gravitation, without the need to postulate invisible matter.

An intriguing possibility is the straightforward explanation of an always constant light velocity via the information self-image of matter. Such an explanation is entirely rational, as Figure 6 visualizes. Information could control photons towards always constant light velocity. On the other hand imagining how empty space around a moving object could adjust light velocity of an arriving photon for constancy within the general theory of relativity is nearly impossible. Since information on matter is simultaneously identified as the phenomenon of gravitation this opens obviously an alternative to the theory of relativity. In fact, when searching for an explanation for an always constant light velocity more than one century ago, a natural information technology should have been taken in consideration. Information can communicate a constant velocity (Figure 6). However, at that time the foundations of information were still unclear with the consequence that they practically do not enter into our understanding of physical laws. Today however the benefits and achievements of artificial information technology are dramatically expanding, and they are working on the basis of existing natural laws. Why should nature itself not use information technology on the basis of gravitation? Of course much has still to be learned and the concept of an information self-image of matter with its consequences, introduced here, may make a beginning.

Even though general relativity theory is now entirely established the author claims that the here suggested approach, to understand gravitation and constant light velocity via the information self-image of matter is much simpler, entirely logic and intuitively understandable. Relativity theory will remain an admirable product of human creativity, but with two significant drawbacks: 1) all crucial physical properties, constant light velocity, gravitation and inertia are designed to be properties of space. However nobody can explain how this works in an environment, the space, originally claimed to contain nothing. The adopted counterintuitive four-dimensional space-time is the consequence; 2) the time in its "crude" form, as the clock shows (in the words of Einstein), is used to describe space-time and its impressive phenomena. It is however only a parameter for monitoring change without energy or substance as basis for measurement. The crude parameter time cannot be used to describe action. In fact, action has to be activated to measure time on the reference system. Properties of energy converting systems have actually to be described by the flow of action, energy times time, as the principle of least action requires. If this is done, if action is transformed and time then locally extracted from action, time dilation and time travel however simply disappear. Action is invariant against transformation.

Gravitation has been recognized as information on matter. Since gravitation is decisive for the dynamics of 
the universe, it may be concluded that it is information, which determines its origin and fate. New cosmological concepts are imaginable, such as the Self-Image universe (Figure 4(b)), which is proposed as a counterpart to the Big Bang scenario (compare also a discussion in [10]). It avoids irrationalities such as creation of energy and time from nothing in a tiny seed, inflation of empty space, an accelerated expansion of the universe and its final end in darkness.

The self-image universe (Figure 4(b)) is created like an object in a 3D printer, energy is conserved, time flow, which here has to be deduced from action, is not interrupted, and the age of the present universe may remain the same when counted from its information mediated recreation.

A remarkable consequence of the proposed paradigm change towards irreversibility in fundamental physics is that many areas of our present physical understanding are affected. A simplified picture of changes, which the irreversibility approach to physical understanding would introduce, is given with the scheme shown in Figure 8. In the center of this figure it is indicated how a study of the meaning of the principle of least action (area with dotted border) led to the concept of a fundamentally dynamic energy and, considering the role of space for energy as a necessary concession for entropy formation, to the consideration of the information self-image of matter for the dynamic particle-wave dualism. They (three darker areas in Figure 8) became the new "symbolic form” (intellectual aid or logical tool), for understanding physical contexts, according to philosopher Ernst Cassirer [45].

As already shown [1] quantization, the double slit experiment and quantum correlation can rationally be understood with the help of the information self-image of matter (left side of Figure 8). In addition it explains gravitation and the constant light velocity with consequences in eliminating relativistic paradoxes and emphasizing the role of information for understanding cosmology (right side and bottom of Figure 8).

With the new understanding of gravitation as a natural form of information another quantum phenomenon can rationally be understood, the tunneling phenomenon (left side of Figure 8). Gravitation is known to penetrate matter and it corresponds to the information self-image of matter in relation (2). It should therefore be able to penetrate barriers with the possibility of resynthesizing the particle with certain probability again on the opposite site. The identification of information on matter with gravitation thus rationally explains the tunneling effect.

In Figure 8, top left, it is indicated how dynamic energy and thus dynamic time allows self-organization of matter, energy and information. Also the information self-image of matter, gravitation, can self-organize under appropriate conditions. The resulting super-gravitation could replace the hypothetical dark matter without need to identify non-visible matter. On the right side of Figure 8 it is visualized that in a dynamic energy world photons can tolerate entropy generation while spreading out in space and generating the microwave background. The Big Bang concept, inflation, the redshift via space expansion, and dark energy concepts are consequently challenged. This concerns also relativity theory, which has been adapted to describe part of these phenomena. The here proposed alternative explanation, deducible for a fundamentally oriented world, basically involves information, a natural phenomenon of information. It is information on matter, which is around matter and could be identified with gravitation.

Up to the present time information is not handled on the basis of recognized fundamental physical laws. However science has learned to deal with information mathematically and in the field of applications we are now witnessing a real revolution of information technology. Since we are using natural laws when dealing with modern information technology, it is claimed here that nature can deal with information and applies it herself because of its incredibly elegant possibilities. The paradigm change towards a fundamentally irreversible world has automatically conduced us to a natural environment controlled and dominated by information, information on matter. This is credible because we are presently learning about the amazing practical possibilities of information for technology.

Science philosopher Karl Popper [46] insisted that a new hypothesis has to suggest the conditions, under which it can be falsified, proven to be incorrect, before being named a new theory. Three falsification conditions can immediately be named for the here advanced arguments for a fundamentally irreversible world: The here proposed concept of a fundamentally irreversible world is incorrect,

1) if it can be shown that a natural process, how simple it may be, can entirely be reversed in time;

2) if it can be shown that significantly diluted energy maintains an identical working ability as concentrated energy;

3) if the presently used statistical time arrow can be derived from time invertible physics without mathematically throwing away information (energy). 


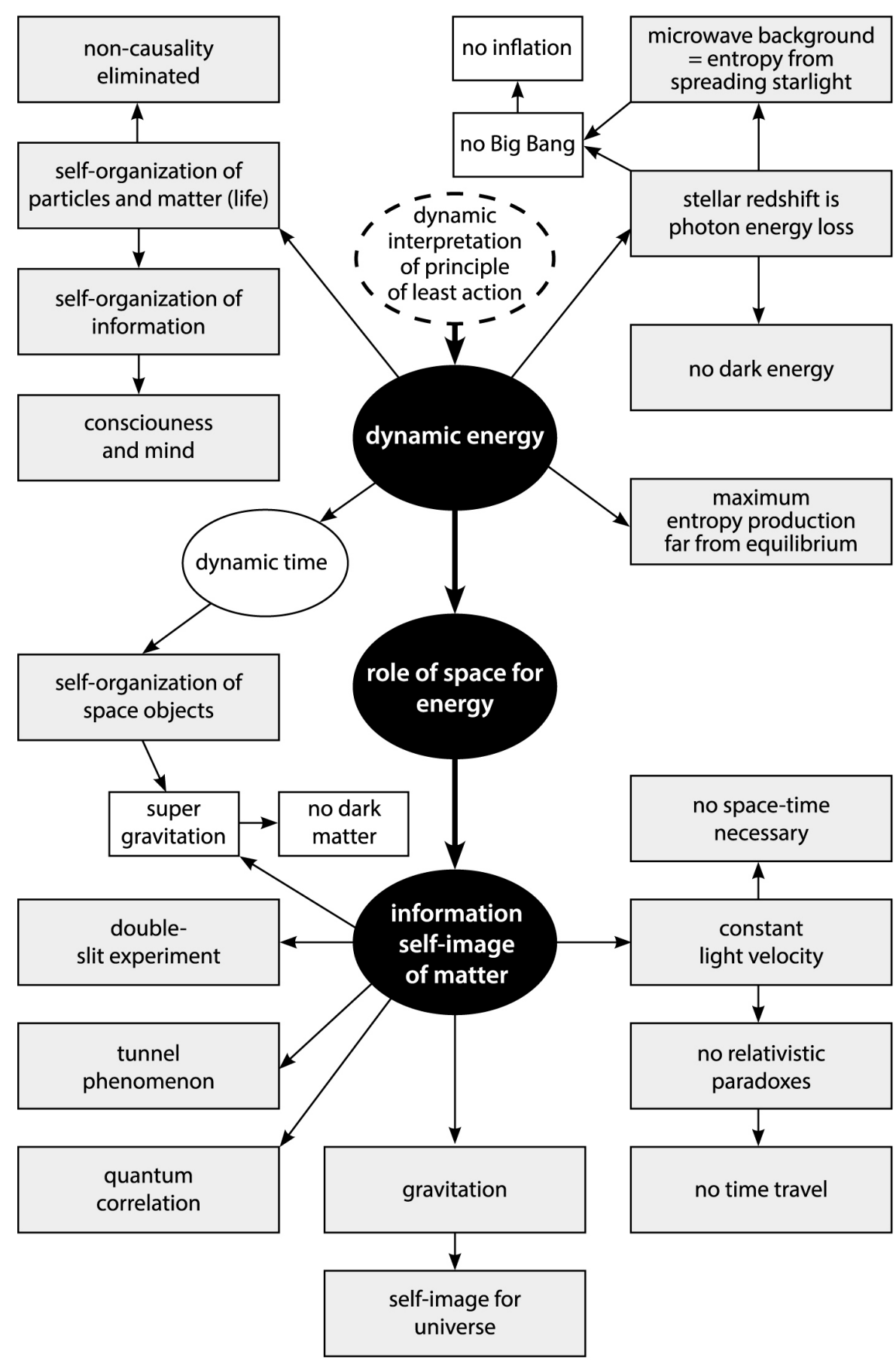

Figure 8. The new "symbolic form”, the intellectual program for understanding physics is a fundamentally dynamic energy-time world, a world of action. It is respecting the role of space for energy and considering an information self-image of matter (three basic claims on dark background in diagram). It is shown how from these derived three basic postulates essential conclusions could be deduced for quantum and space phenomena and contexts.

Under such conditions one can talk of a new theory, the theory of a fundamental oriented and irreversible world. In turn, when above conditions 1) to 3) cannot be fulfilled, this is strong evidence against a fundamentally time-invertible world, as it is presently sustained in physics.

The fact that essentially one single paradigm change, the claim of fundamental orientation and irreversibility 
in nature, even derivable from the principle of least action [1], can eliminate well known and perturbing paradoxes of physics, deserves attention. And the finding that such a small (but significant) change can yield a remarkably different view of the universe should induce modesty with respect to our present scientific understanding of nature. It is anyway remarkable that the concept of a fundamentally time invertible physics with all its resulting paradoxes and irrationalities could become so powerful over such a long time, even though everything is changing in the universe and nothing actually proved to be entirely time invertible.

After all, it is well known that the Franciscan friar William of Ockham from the 14th century suggested that "one should not admit more causes of natural things than is sufficient to explain them". This rule of thumb for scientists, also known as Ockham's razor, would clearly favour the "dynamic" energy approach presented here since it is essentially based on a single paradigm change with the consequence that essential paradoxes, irrationalities and deficiencies can be eliminated or questioned (quantum paradoxes, action without cause, fundamental uncertainty, four-dimensional space-time concept, time travel, inflation of empty space, dark matter, dark energy, inability to explain consciousness and spirit materialistically). Also entropy laws for reversible and irreversible thermodynamics can be logically derived. The inability to do that is a significant drawback for present physical understanding within a time-invertible fundamental physical nature.

The concept proposed here is a working frame, which, of course, has to be deepened in nearly every respect. Only time (flow of action) can facilitate that. It promises a drastically simplified and rational understanding of nature, though with significant new challenges. The natural matter-related information system, looked upon as gravitation, which links quantum processes with cosmological phenomena, needs to be deciphered and self-organization of information explored. We may learn, that also nature around us has grown via a revolution of information technology and that an information based, much more logic and intelligent universe, the Self-Image universe”, may replace the Big Bang scenario (compare also [10]).

And we may finally answer a question, which, beginning with Heraklitus of Ephesus in the $6^{\text {th }}$ century BC, ancient natural philosophers have already been asking. They saw that everything in their environment was changing and were wondered what may be conserved (and still be related to the observed changes) [47] [48]. Now we could answer saying: it is energy with its tendency to decrease its presence per state (relation (1)). Energy is conserved but simultaneously deeply involved in changes. In contrast, the conventional, presently applied energy concept, energy as a scalar quantity, with the ability but no interest to do work, did not conserve an obvious relation to change. It somehow forgot the point of departure, from where the search for the energy concept originally started. It became therefore, in some way, a dead end road, facing more and more paradoxes and irrationalities. The here presented concept of a fundamentally oriented world offers an alternative open road to be explored.

\section{References}

[1] Tributsch, H. (2016) Journal of Modern Physics, 7, 365-374. http://dx.doi.org/10.4236/jmp.2016.74037

[2] Einstein, A. (1905) Annalen der Physik, 17, 132-148. http://dx.doi.org/10.1002/andp.19053220607

[3] Darrigol, O. (1897) Planck’s Radiation Theory. Chapter III, on Irreversible Radiation Processes. UC Press, Berkeley. http://publishing.cdlib.org/ucpressebooks/view?docId=ft4t1nb2gv\&chunk.id=d0e2674\&toc.depth=1\&brand=ucpress

[4] Kelly, R.E. (1980) American Journal of Physics, 49, 714-719. http://dx.doi.org/10.1119/1.12416

[5] Smoot, G.F. (2006) Cosmic Microwave Background Radiation Anisotropies: Their Discovery and Utilization. Nobel Lecture, Nobel Foundation.

[6] Guth, A.H. (1998) The Inflationary Universe: The Quest for a New Theory of Cosmic Origins. Basic Books, New York.

[7] Bell, J.S. (1964) Physics, 1, 195-200.

[8] Clauser, J.F. and Shimony, A. (1978) Reports on Progress in Physics, 41, 1881. http://dx.doi.org/10.1088/0034-4885/41/12/002

[9] Gödel, K. (2016). https://en.wikipedia.org/wiki

[10] Tributsch, H. (2015) Irrationality in Nature or in Science? Probing a Rational Energy and Mind World. CreateSpace, a Company of Amazon.

Tributsch, H. (2016) Der logische Schlüssel zum Universum: Zeit, Information und die Dynamik von Energie, Quanten und Geist. CreateSpace, a Company of Amazon. (To Be Published)

[11] Microwave Background Radiation (2016). 
https://en.wikipedia.org/wiki/Cosmic_microwave_background

[12] Ritz, W. (1908) Annales de Chimie et de Physique, 8, 145.

[13] Zwicky, F. (1929) Proceedings of the National Academy of Sciences, 15, 773-779. http://dx.doi.org/10.1073/pnas.15.10.773

[14] Berliner, L.M. (1992) Statistical Science, 7, 69-90. http://dx.doi.org/10.1214/ss/1177011444

[15] Rugh, S.E. and Zinkernagel, H. (2000) The Quantum Vacuum and the Cosmological Constant Problem. http://arxiv.org/pdf/hep-th/0012253.pdf

[16] Paltridge, G.W. (1979) Nature, 279, 630-631. http://dx.doi.org/10.1038/279630a0

[17] Liu, Y., Liu, C. and Wang, D. (2011) Entropy, 13, 211-240. http://dx.doi.org/10.3390/e13010211

[18] Swenson, R. (1997) Advances in Human Ecology, 6, 1-47.http://rodswenson.com/humaneco.pdf

[19] Ross, J. (2008) Thermodynamics and Fluctuations Far From Equilibrium (12.5: Invalidity of the Principle of Maximum Entropy Production). Springer, Berlin, 119.

[20] Kohaut, E. and Weiss, W. (2007) Das Rätsel Gravitation. Edition Va Bene.

[21] GUT (2016). https://en.wikipedia.org/wiki/Grand_Unified_Theory

[22] Einstein, A. (1920) Ether and Theory of Relativity. Lecture Given at the University of Leiden on 5th May, 1920.

[23] Gravitational Waves Detected (2016).

http://www.sciencealert.com/live-update-big-gravitational-wave-announcement-is-happening-right-now

[24] Big Bang Criticism (2016). http://thetechreader.com/top-ten/top-ten-scientific-flaws-in-the-big-bang-theory

[25] Bible (Old Testament) (2nd to 1st Millennium BC, Compiled 450 BC) John 1:1.

[26] Nagel, T. (2012) Mind and Cosmos, Why the Materialist Neo-Darwinian Conception of Nature Is Almost Certainly False. Oxford University Press, Oxford. http://dx.doi.org/10.1093/acprof:oso/9780199919758.001.0001

[27] Aether (2016). https://en.wikipedia.org/wiki/Aether_theories

[28] Lichnerowicz, A. (1955) Theories relativistes de la gravitation et de l'electromagnetisme. Masson et Cie, Paris.

[29] General Relativity (2016). http://en.wikipedia.org/wiki/Mathematics_of_general_relativity

[30] Ives, H.E. (1952) Journal of the Optical Society of America, 42, 540-543. http://dx.doi.org/10.1364/josa.42.000540

[31] Gut, B.J. (1981) Immanent-logische Kritik der Relativitätstheorie. Oberwil b. Kugler, Zug, 151 S.

[32] De Pretto, O. (1903). http://en.wikipedia.org/wiki/Olinto_De_Pretto

[33] Hawking, S. (2016) Space and Time Warps. http://www.hawking.org.uk/space-and-time-warps.html

[34] Augustine, S. (2012) Confessions. Simon \& Brown, Los Angeles.

[35] Reichenbach, H. (1999) The Direction of Time. Dover, New York.

[36] Whitrow, G.J. (1988) Time in History. The Evolution of Our General Awareness of Time and Temporal Perspective. Oxford University Press, Oxford.

[37] Rovelli, C. (2006) What Is Time? What Is Space? Di Renzo Editore, Rome.

[38] Heidegger, M. (1962) “V”. Being and Time. Blackwell, Oxford, UK \& Cambridge, USA, 425.

[39] Davies, P. (1996) About Time: Einstein’s Unfinished Revolution. Simon \& Schuster Paperbacks, New York.

[40] Tributsch, H. (2008) Energy, Time and Consciousness. Shaker Media, Aachen.

[41] Time (2016) Wikipedia. https://en.wikipedia.org/wiki/Time

[42] Hawking Radiation (2016). https://en.wikipedia.org/wiki/Hawking_radiation

[43] Black Hole (2016). https://en.wikipedia.org/wiki/Black_hole

[44] Quasars (2016) https://en.wikipedia.org/wiki/Quasar

[45] Cassirer, E. (1923) Philosophie der symbolischen Formen, Die Sprache, Bd. 1; Das mythische Denken (1924), Bd. 2; Phänomenologie der Erkenntnis (1929), Bd. 3; Darmstadt (1997).

[46] Popper, K.R. (1979) Die beiden Grundprobleme der Erkenntnistheorie (Herausgeber: Troels Eggers Hansen), J.C.B. Mohr (Paul Siebeck) Tübingen, 426-427.

[47] Lindsay, R.B. (1971) Foundations of Physics, 1, 383-393. http://dx.doi.org/10.1007/BF00708586

[48] Lindsay, R.B., Ed. (1975) Energy: Historical Development of the Concept. Benchmark Papers on Energy, v. 1, Dowden, Hutchingson \& Ross, Inc., Stroudsburg. 


\section{Submit or recommend next manuscript to SCIRP and we will provide best service for you:}

Accepting pre-submission inquiries through Email, Facebook, LinkedIn, Twitter, etc.

A wide selection of journals (inclusive of 9 subjects, more than 200 journals)

Providing 24-hour high-quality service

User-friendly online submission system

Fair and swift peer-review system

Efficient typesetting and proofreading procedure

Display of the result of downloads and visits, as well as the number of cited articles

Maximum dissemination of your research work

Submit your manuscript at: http://papersubmission.scirp.org/ 